TRANSACTIONS OF THE

AMERICAN MATHEMATICAL SOCIETY

Volume 361, Number 1, January 2009, Pages 267-295

S 0002-9947(08)04477-2

Article electronically published on August 14, 2008

\title{
BANACH ALGEBRAS AND RATIONAL HOMOTOPY THEORY
}

\author{
GREGORY LUPTON, N. CHRISTOPHER PHILLIPS, CLAUDE L. SCHOCHET, \\ AND SAMUEL B. SMITH
}

\begin{abstract}
Let $A$ be a unital commutative Banach algebra with maximal ideal space $\operatorname{Max}(A)$. We determine the rational $\mathrm{H}$-type of $\mathrm{GL}_{n}(A)$, the group of invertible $n \times n$ matrices with coefficients in $A$, in terms of the rational cohomology of $\operatorname{Max}(A)$. We also address an old problem of J. L. Taylor. Let $\mathrm{Lc}_{n}(A)$ denote the space of "last columns" of $\mathrm{GL}_{n}(A)$. We construct a natural isomorphism

$$
\check{H}^{s}(\operatorname{Max}(A) ; \mathbb{Q}) \cong \pi_{2 n-1-s}\left(\operatorname{Lc}_{n}(A)\right) \otimes \mathbb{Q}
$$

for $n>\frac{1}{2} s+1$ which shows that the rational cohomology groups of $\operatorname{Max}(A)$ are determined by a topological invariant associated to $A$. As part of our analysis, we determine the rational H-type of certain gauge groups $F(X, G)$ for $G$ a Lie group or, more generally, a rational H-space.
\end{abstract}

\section{INTRODUCTION}

Let $A$ be a unital commutative Banach algebra. $\operatorname{By} \operatorname{Max}(A)$ we denote the set of maximal ideals of the Banach algebra $A$ topologized with the relative weak*topology. Let $\mathrm{GL}_{n}(A)$ denote the group of invertible $n \times n$ matrices with coefficients in $A$. In this paper, we describe the rational homotopy of $\mathrm{GL}_{n}(A)$ and also that of a second topological space associated to $A$.

The difficulty inherent in describing the ordinary homotopy theory of the group $\mathrm{GL}_{n}(A)$ is apparent even in the simplest cases. For instance, when $A=\mathbb{C}$ we have

$$
\pi_{j}\left(\mathrm{GL}_{2}(\mathbb{C})\right) \cong \pi_{j}\left(S^{3}\right)
$$

for $j>1$. Now $\pi_{j}\left(S^{3}\right) \neq 0$ for infinitely many values of $j$, and these groups are known only for $j<100$ or so. Of course this particular problem becomes tractable after rationalization, since J.-P. Serre showed that $\pi_{j}\left(S^{3}\right)$ is finite for $j>3$.

In fact, substantially more can be said about $\mathrm{GL}_{n}(\mathbb{C})$ rationally, using classical results and methods. For instance, observe that polar decomposition provides a canonical deformation retraction of $\mathrm{GL}_{n}(\mathbb{C})$ to the compact Lie group $U_{n}(\mathbb{C})$. By results going back to $\mathrm{H}$. Hopf, the rational cohomology algebra of $U_{n}(\mathbb{C})$ is an exterior algebra generated in odd degrees $1,3, \ldots, 2 n-1$. From these facts, we deduce a rational homotopy equivalence

$$
\mathrm{GL}_{n}(\mathbb{C}) \simeq_{\mathbb{Q}} S^{1} \times S^{3} \times \cdots \times S^{2 n-1} .
$$

Received by the editors April 17, 2006 and, in revised form, December 19, 2006.

2000 Mathematics Subject Classification. Primary 46J05, 46L85, 55P62, 54C35, 55P15, 55P45.

Key words and phrases. Commutative Banach algebra, maximal ideal space, general linear group, space of last columns, rational homotopy theory, function space, rational H-space, gauge groups.

The research of the second author was partially supported by NSF grant DMS 0302401.

(C)2008 American Mathematical Society Reverts to public domain 28 years from publication 
(See Example 4.16 below.) This equivalence actually determines the structure of $\mathrm{GL}_{n}(\mathbb{C})$ as a rational H-space since a homotopy-associative H-space with only odd rational homotopy groups is rationally homotopy-abelian. (See Corollary 4.27)

Our first main result extends the above analysis to the general case. We determine the rational homotopy type of $\mathrm{GL}_{n}(A)$ in terms of the rational Cech cohomology groups $\check{H}^{*}(\operatorname{Max}(A) ; \mathbb{Q})$ of the space $\operatorname{Max}(A)$. In fact, we determine the full structure of $\mathrm{GL}_{n}(A)$ as a rational H-space. To describe our results, we introduce some notation.

Given a space $X$ with basepoint, we write $X_{\circ}$ for the path component of the basepoint of $X$. Recall that if $\pi$ is an abelian group, then $K(\pi, n)$ is the corresponding Eilenberg-Mac Lane space. (See Example 4.5.) If $\pi$ is countable abelian, by a result of J. Milnor [20] (also see Milgram [19]) multiplication of loops gives $K(\pi, n)$ the structure of an abelian topological group, and this structure is unique up to homotopy. (Again, see Example 4.5.) Consequently, a product $\prod_{j>1} K\left(\pi_{j}, j\right)$ of Eilenberg-Mac Lane spaces with its standard loop multiplication also has the structure of an abelian topological group. (This multiplication is rarely unique, even up to homotopy. See Example 4.6.)

Our first main result is the following.

Theorem 1. Suppose that $A$ is a unital commutative Banach algebra with maximal ideal space $\operatorname{Max}(A)$. Let

$$
\check{V}_{h, j}=\check{H}^{2 j-1-h}(\operatorname{Max}(A) ; \mathbb{Q}) .
$$

Then there is a natural rational H-equivalence

$$
\mathrm{GL}_{n}(A)_{\circ} \simeq \mathbb{Q} \prod_{j=1}^{n} \prod_{h=1}^{2 j-1} K\left(\check{V}_{h, j}, h\right)
$$

where the product of Eilenberg-Mac Lane spaces has the standard loop multiplication. Thus $\mathrm{GL}_{n}(A)$ 。 has the rational H-type of an abelian topological group.

As a consequence of Theorem 1] we address an old question of J. L. Taylor regarding the cohomology of the space $\operatorname{Max}(A)$. In [29], Taylor raised the question of characterizing topological invariants of $\operatorname{Max}(A)$ in terms of invariants of the algebra. Taylor showed that classical results give a way to characterize $H^{n}(\operatorname{Max}(A) ; \mathbb{Z})$ for $n=0,1,2$. To be more specific:

- $H^{0}(\operatorname{Max}(A) ; \mathbb{Z})$ consists of formal combinations of idempotents of $A$. Taylor derives this from the Shilov Idempotent Theorem.

- $H^{1}(\operatorname{Max}(A) ; \mathbb{Z})$ is the quotient of $\operatorname{GL}(A)$ by the image of the exponential map exp: $A \rightarrow \mathrm{GL}(A)$. Taylor derives this from the Arens-Royden Theorem. For $A=C(X)$, the algebra of continuous complex valued functions on a compact Hausdorff space, and when $X$ is metrizable, K. Thomsen observes (see the introduction to Section 4 of [32]) that this is a (1934) theorem of Bruschlinsky.

- $H^{2}(\operatorname{Max}(A) ; \mathbb{Z})$ is the Picard group $\operatorname{Pic}(A)$ of $A$ by a result of Forster. The Picard group consists of isomorphism classes of finitely generated projective $A$-modules $P$ which are invertible in the sense that there exists some finitely generated projective module $Q$ such that $P \otimes Q$ is a free module of rank 1 over $A$. For a different, and deeper, version of the Picard group, see [6]. 
We note that these results are easy in the case $A=C(X)$; the main point is that they may be extended to commutative Banach algebras.

In addition, we have the result of J. Dixmier and A. Douady [11]:

- $H^{3}(X ; \mathbb{Z})$ is isomorphic to the Brauer group of algebra bundles over $X$ with fibre $\mathcal{K}$, the compact operators on a separable, infinite-dimensional Hilbert space.

As far as we know, there has been no further progress describing $H^{n}(\operatorname{Max}(A) ; \mathbb{Z})$ in a concrete fashion. However, the recent interesting work of A. B. Thom [30] does shed additional light on the problem, albeit in a very abstract context.

As regards $K$-theory, we can add the following results, which were known to Atiyah, Adams, Swan and others:

- $K^{0}(\operatorname{Max}(A))$ is isomorphic to $K_{0}(A)$, which is defined (for any ring $A$ ) as the quotient of the free abelian group generated by all finitely generated projective left $A$-modules modulo the isomorphism and direct sum relations.

- $K^{1}(\operatorname{Max}(A))$ is isomorphic to $\mathrm{GL}_{\infty}(A) / \mathrm{GL}_{\infty}(A)_{\circ}$, the quotient of the infinite general linear group of $A$ by the connected component of the identity.

Now consider the natural action of $\mathrm{GL}_{n}(A)$ on the right $A$-module $A^{n}$ (the direct sum of $n$ copies of $A$ ). The space of "last columns" $\operatorname{Lc}_{n}(A)$ is defined to be the orbit of the last standard basis vector $e_{n} \in A^{n}$ under the action of $\mathrm{GL}_{n}(A)$. This is a familiar space, studied for example in 25. With our second main result, we provide an answer to Taylor's question for the rational (Čech) cohomology groups of the space $\operatorname{Max}(A)$.

Theorem 2. Let $A$ be a unital commutative Banach algebra. Then the rational cohomology groups of $\operatorname{Max}(A)$ are determined by a topological invariant of $A$ via a natural isomorphism

$$
\check{H}^{s}(\operatorname{Max}(A) ; \mathbb{Q}) \cong \pi_{2 n-1-s}\left(\operatorname{Lc}_{n}(A)\right) \otimes \mathbb{Q}
$$

for $n>\frac{1}{2} s+1$.

In an effort to make the paper understandable to both Banach algebraists and algebraic topologists, we include more details than would be needed in a paper aimed at either audience alone. The organization is as follows. In Section 2 we introduce the topological spaces associated to a Banach algebra that we will need. In Section 3 we begin our examination of the homotopy groups of the general linear groups of the commutative Banach algebras. The group $\mathrm{GL}_{n}(A)$ is known to be homotopy equivalent to the space of continuous functions $F\left(\operatorname{Max}(A), \mathrm{GL}_{n}(\mathbb{C})\right)$ by an important result of Davie [10. We reduce the problem of identifying $\operatorname{Lc}_{n}(A)$ to one of studying the path component of the constant map in the function space $F\left(\operatorname{Max}(A), S^{2 n-1}\right)$ (Theorem 3.6) . Consequently, we are concerned with studying function spaces of the form $F(X, G)$, for $G$ a topological group or, more generally, of the rational homotopy type of a topological group. In Section 4 we develop some ideas, mostly from rational homotopy theory, that are sufficient to completely understand the rational homotopy type of a function space of this latter form when $X$ and $G$ are finite complexes. The results of this section are developed in greater generality than is strictly necessary, and some are of independent interest for the 
homotopy of function spaces (e.g. Theorem 4.10). Finally, in Section 5, we prove Theorems 1 and 2 by applying the results developed in Section 4

For any topological spaces $X$ and $Y$ we let $F(X, Y)$ denote the set of continuous functions $X \rightarrow Y$ with the compactly generated topology. (See Section IV.3 of [33].) This topology agrees with the compact-open topology on compact sets. Hence using this topology rather than the compact-open topology has no effect on homotopy and singular homology. If $X$ and $Y$ are based spaces, then we let $F_{\bullet}(X, Y)$ denote the subspace of basepoint-preserving maps. We use $[X, Y]$ to denote the set of based homotopy classes of based maps from $X$ to $Y$. When working with a topological group we take the basepoint to be the identity of the group. Basepoints are always assumed to be non-degenerate, that is, the inclusion of the basepoint into the space is a cofibration, or equivalently, the pair consisting of the space and its basepoint is an NDR-pair. (See page 7 and Section I.4 of [33.) Observe that $f: X \rightarrow Y$ induces maps $f_{*}: F(W, X) \rightarrow F(W, Y)$ and $f^{*}: F(Y, Z) \rightarrow F(X, Z)$ given by composition and pre-composition with $f$, respectively. We also use this notation for the maps induced by $f$ on basepoint-preserving function spaces and on sets of homotopy classes of maps.

A function space is typically not path connected. We take the basepoint of $F(X, Y)$ and $F_{\bullet}(X, Y)$ to be the constant map sending $X$ to the basepoint of to $Y$. Thus $F(X, Y)$ o denotes the space of maps from $X$ to $Y$ which are freely homotopic to the constant map. Similarly, $F_{\bullet}(X, Y)$ 。 denotes the space of based, null-homotopic maps. If $G$ is a topological group, then the function space $F(X, G)$ is one also, with multiplication given by pointwise multiplication of maps. In this case, the basepoint is the constant map carrying $X$ to the identity of $G$. For any compact space $X$ we let $C(X)$ denote the $C^{*}$-algebra of continuous complex-valued functions on $X$ with the operations given pointwise. We denote by $M_{n}(A)$ the algebra of $n \times n$ matrices with entries in $A$. If $A$ is a Banach algebra, then $M_{n}(A)$ is also a Banach algebra in an obvious way.

\section{The SPACES}

In this section, we let $A$ be any Banach algebra. While later we will be interested exclusively in the case where $A$ is commutative and unital, the results of this section can be stated in somewhat greater generality. We follow Corach and Larotonda [7, [8] and Rieffel [25] in discussing the spaces associated to $A$ that we will need. Following Section 3 of [32, we handle non-unital algebras by unitizing them and making slight modifications of the definitions. In order to preserve functoriality, we therefore unitize all algebras. Thus, the definitions of $\mathrm{GL}_{n}(A)$ and $\mathrm{Lc}_{n}(A)$ given below do not agree with those in the Introduction. We will show in Proposition 2.11 below that, when $A$ is unital, they are nevertheless equivalent.

Notation 2.1. If $A$ is a Banach algebra, then we denote by $A^{+}$its unitization $A \oplus \mathbb{C}$, with the elements written $a+\lambda \cdot 1$ (or just $a+\lambda$ ) for $a \in A$ and $\lambda \in \mathbb{C}$, and the multiplication obtained by applying the distributive law in the obvious way. (See Definition 1 in Section 3 of [4], or the end of Section 1.2 of [22].)

The unitization is again a Banach algebra. If $A$ is a $C^{*}$-algebra, then there is a unique choice of norm making $A^{+}$a $C^{*}$-algebra (Theorem 2.1.6 of [22]).

Remark 2.2. When forming $A^{+}$, we add a new identity even if $A$ is already unital. In this case, $A^{+}$is isomorphic to the Banach algebra direct sum $A \oplus \mathbb{C}$. Letting $1_{A}$ 
and $1_{A^{+}}$denote the identities of $A$ and $A^{+}$, the isomorphism is given by

$$
a+\lambda \cdot 1_{A^{+}} \mapsto\left(a+\lambda \cdot 1_{A}, \lambda\right) .
$$

This map is a homeomorphism, and if $A$ is a $C^{*}$-algebra it is an isometry.

Definition 2.3. Let $A$ be a Banach algebra and let $A^{+}$denote its unitization as in Notation 2.1, We set

$$
\mathrm{GL}_{n}(A)=\left\{a \in M_{n}\left(A^{+}\right): a \text { is invertible and } a-1 \in M_{n}(A)\right\},
$$

with the relative topology from $M_{n}\left(A^{+}\right)$.

Note that $M_{n}(A)$ is a subset of $M_{n}\left(A^{+}\right)$in an obvious way, and that, in this definition, 1 denotes the identity of $M_{n}\left(A^{+}\right)$.

Remark 2.4. Let $A$ be a Banach algebra. For $a=\left(a_{j, k}\right) \in \operatorname{GL}_{n}(A)$ and $x=$ $\left(x_{1}, \ldots, x_{n}\right) \in\left(A^{+}\right)^{n}$, define $a x$ to be the usual left action of $a \in M_{n}\left(A^{+}\right)$, the right $A^{+}$-module endomorphisms of $\left(A^{+}\right)^{n}$ when elements of $\left(A^{+}\right)^{n}$ are viewed as column vectors. This defines a jointly continuous left action of $\operatorname{GL}_{n}(A)$ on $\left(A^{+}\right)^{n}$.

Definition 2.5. Let $A$ be a Banach algebra. We define

$$
\begin{aligned}
\operatorname{Lg}_{n}(A)=\left\{\left(x_{1}, \ldots, x_{n}\right) \in\left(A^{+}\right)^{n}: x_{1}, \ldots, x_{n-1}, x_{n}-1 \in A\right. \text { and } \\
\text { there are } \left.y_{1}, \ldots, y_{n} \in A^{+} \text {such that } \sum_{k=1}^{n} y_{k} x_{k}=1\right\} .
\end{aligned}
$$

We define $\operatorname{Lc}_{n}(A)$ to be the orbit of the last standard basis vector $e_{n}=(0, \ldots, 0,1) \in$ $\left(A^{+}\right)^{n}$ under the action of $\mathrm{GL}_{n}(A)$ on $\left(A^{+}\right)^{n}$ of Remark 2.4. We let $\gamma: \mathrm{GL}_{n}(A) \rightarrow$ $\operatorname{Lc}_{n}(A)$ (or $\gamma_{A}$ if it is necessary to specify the algebra) be $\gamma(a)=a \cdot e_{n}$. Finally, we define $\mathrm{TL}_{n}(A) \subset \mathrm{GL}_{n}(A)$ to be $\left\{a \in G L_{n}(A): a \cdot e_{n}=e_{n}\right\}$. All these spaces are given the relative norm topology from $\left(A^{+}\right)^{n}$ or $M_{n}\left(A^{+}\right)$as appropriate.

As we will see in Proposition 2.11, the definitions of $\mathrm{GL}_{n}(A)$ and $\mathrm{Lc}_{n}(A)$ simplify to those given in the Introduction when $A$ is unital.

Remark 2.6. One immediately checks that $\mathrm{TL}_{n}(A)$ is the subgroup of $\mathrm{GL}_{n}(A)$ consisting of matrices of the form

$$
\left[\begin{array}{ll}
x & 0 \\
c & 1
\end{array}\right],
$$

where $x \in \mathrm{GL}_{n-1}(A)$ and $c$ is any row of elements of $A$ of length $n-1$.

Lemma 2.7. Let $A$ be a Banach algebra.

(1) The closed vector subspace

$$
L_{n}=\left\{x=\left(x_{1}, \ldots, x_{n}\right) \in\left(A^{+}\right)^{n}: x-e_{n} \in A^{n}\right\}
$$

is invariant under the action of $\mathrm{GL}_{n}(A)$.

(2) The space $\operatorname{Lg}_{n}(A)$ is invariant under the action of $\mathrm{GL}_{n}(A)$.

(3) We have $\operatorname{Lc}_{n}(A) \subset \operatorname{Lg}_{n}(A)$, and $\operatorname{Lc}_{n}(A)$ is equal to the set of all $n$-tuples of elements of $A^{+}$which occur as the last column of an element of $\mathrm{GL}_{n}(A)$.

Proof. We prove Part (11). Let $a \in \mathrm{GL}_{n}(A)$ and let $x \in L_{n}$. Then $a-1 \in M_{n}(A)$, from which it easily follows that $(a-1) x \in A^{n}$. Also $x-e_{n} \in A^{n}$. So

$$
a x-e_{n}=(a-1) x+\left(x-e_{n}\right) \in A^{n} .
$$

For Part (2), let $a \in \mathrm{GL}_{n}(A)$ and let $x \in \operatorname{Lg}_{n}(A)$. Then $a \cdot x-e_{n} \in A^{n}$ by Part (1). Choose $y=\left(y_{1}, \ldots, y_{n}\right) \in\left(A^{+}\right)^{n}$ such that $y_{1} x_{1}+\cdots+y_{n} x_{n}=1$. Regarding $y$ as 
a row vector, form the matrix product $z=y \cdot a^{-1} \in\left(A^{+}\right)^{n}$. Then one checks that $\sum_{k=1}^{n} z_{k}(a \cdot x)_{k}=1$; so $a \cdot x \in \operatorname{Lg}_{n}(A)$.

We prove Part (3) . To identify $\operatorname{Lc}_{n}(A)$ as the space of last columns, simply observe that $a \cdot e_{n}=x$ if and only if $\left(x_{1}, \ldots, x_{n}\right)$ is the last column of $a$. The first statement follows from this observation and (2).

The following theorem is primarily due to Corach and Larotonda (Theorem 1 of [7]) in the unital case, with parts due to Thomsen (Section 3 of [32]) in the $C^{*}$-algebra case. We will follow 32 for most of the proof, since it uses machinery more familiar in Banach algebras.

Theorem 2.8. Let $A$ be a Banach algebra. Then:

(1) For each $x \in \operatorname{Lg}_{n}(A)$, the map $a \mapsto a \cdot x$ is an open mapping (not surjective) from $\mathrm{GL}_{n}(A)$ to

$$
L_{n}=\left\{x=\left(x_{1}, \ldots, x_{n}\right) \in\left(A^{+}\right)^{n}: x-e_{n} \in A^{n}\right\} .
$$

(2) The map $\gamma$ defines a principal locally trivial fibre bundle with structural group $\mathrm{TL}_{n}(A)$.

(3) The sequence

$$
\mathrm{TL}_{n}(A) \longrightarrow \mathrm{GL}_{n}(A) \stackrel{\gamma}{\longrightarrow} \mathrm{Lc}_{n}(A)
$$

is a Serre fibration, with base point preserving maps.

(4) This Serre fibration is natural in A, using the obvious maps (described explicitly in the proof).

(5) The spaces $\mathrm{GL}_{n}(A), \mathrm{TL}_{n}(A)$, and $\mathrm{Lc}_{n}(A)$ are homeomorphic to open subsets of Banach spaces, and they have the homotopy type of $C W$ complexes.

(6) We have $\operatorname{Lg}_{n}(A)_{\circ}=\operatorname{Lc}_{n}(A)_{\circ}$, and the map $\gamma$ restricts to an open map of $\mathrm{GL}_{n}(A)$ 。 onto $\operatorname{Lc}_{n}(A)_{\circ}$.

Proof. For $C^{*}$-algebras (not necessarily unital), Part (1) is a slight strengthening of Lemma 3.3 of [32]. We follow the proof of Theorem 8.3 of [24]. Let $a \in \mathrm{GL}_{n}(A)$ and let $x \in \operatorname{Lg}_{n}(A)$. Set $y=a \cdot x$, and let $\varepsilon>0$. We find $\delta>0$ such that whenever $z \in L_{n}$ and $\left\|z_{k}-y_{k}\right\|<\delta$ for $1 \leq k \leq n$, then there is $b \in \operatorname{GL}_{n}(A)$ such that $\|b-a\|<\varepsilon$ and $b \cdot x=z$. This will clearly suffice to prove Part (1).

Now, because $y \in \operatorname{Lg}_{n}(A)$ (Lemma 2.7(2)), there are $r_{1}, \ldots, r_{n} \in A$ such that $r_{1} y_{1}+\cdots+r_{n} y_{n}=1$. Set

$$
M=\max \left(\left\|r_{1}\right\|, \ldots,\left\|r_{n}\right\|\right),
$$

and choose $\delta>0$ so small that

$$
\delta n^{2} M<1 \quad \text { and } \quad\left(1-\delta n^{2} M\right)^{-1}<\frac{\varepsilon}{\|a\|} .
$$

Let $\left(z_{1}, \ldots, z_{n}\right) \in A^{n}$ satisfy $\left\|z_{k}-y_{k}\right\|<\delta$ for all $k$. Let $c$ be the matrix with the $(j, k)$ entry equal to $\left(z_{j}-y_{j}\right) r_{k}$. Then $c \in M_{n}(A)$ because $z_{j}-y_{j} \in A$ for all $j$. Also,

$$
c_{j, 1} x_{1}+\cdots+c_{j, n} x_{n}=\left(z_{j}-y_{j}\right) r_{1} x_{1}+\cdots+\left(z_{j}-y_{j}\right) r_{n} x_{n}=z_{j}-y_{j} .
$$

Therefore $(1+c) y=z$, whence $b=(1+c) a$ satisfies $b x=z$. Finally,

$$
\|c\| \leq \sum_{j, k=1}^{n}\left\|\left(z_{j}-y_{j}\right) r_{k}\right\|<\delta n^{2} M .
$$


Therefore $1+c$ is invertible in $M_{n}(A)$, with inverse

$$
(1+c)^{-1}=1-c+c^{2}-c^{3}+\cdots .
$$

In particular, $(1+c)^{-1}-1 \in M_{n}(A)$, so $c \in \mathrm{GL}_{n}(A)$, and

$$
\left\|(1+c)^{-1}\right\| \leq(1-\|c\|)^{-1}<\left(1-\delta n^{2} M\right)^{-1}<\frac{\varepsilon}{\|a\|} .
$$

So $b \in \mathrm{GL}_{n}(A)$ and $\|b-a\|<\varepsilon$. This proves Part (1).

For $C^{*}$-algebras, Parts (22) and (3) are in Corollary 3.5 of [32] and the discussion after Lemma 3.7 of 32 (except for the part about the maps preserving the basepoints, which is immediate from our conventions on basepoints), and Part (6) follows from Lemma 3.7 of [32. For Banach algebras, the only required change is in the definition of $\operatorname{Lg}_{n}(A)$; see Definition 2.5.

The second statement in Part (5) for $\operatorname{Lc}_{n}(A)$ is Lemma 3.6 of [32. For Banach algebras, we first recall that, by Corollary 5.5 in Chapter 4 of [18], every open subset of a Banach space has the homotopy type of a CW complex. So it is enough to prove the first statement. For $\operatorname{Lc}_{n}(A)$, Part (11) implies that $\operatorname{Lc}_{n}(A)$ is an open subset of the Banach space $L_{n}$ there. Also, $\operatorname{GL}_{n}(A)$ is homeomorphic to the open subset of $A^{n^{2}}$ consisting of those $\left(a_{j, k}\right)_{j, k=1}^{n} \in A^{n^{2}}$ such that

$$
\left[\begin{array}{cccc}
a_{1,1}+1 & a_{1,2} & \cdots & a_{1, n} \\
a_{2,1} & a_{2,2}+1 & \cdots & a_{2, n} \\
\vdots & \vdots & \ddots & \vdots \\
a_{n, 1} & a_{n, 2} & \cdots & a_{n, n}+1
\end{array}\right]
$$

is invertible in $A^{+}$. Similarly, $\mathrm{TL}_{n}(A)$ is homeomorphic to an open subset of $A^{n(n-1)}$.

Finally, we consider Part (4). Let $A$ and $B$ be Banach algebras, and let $\varphi: A \rightarrow B$ be a continuous homomorphism (not necessarily unital even if $A$ and $B$ are both unital). Then there is a continuous unital homomorphism $\varphi^{+}: A^{+} \rightarrow B^{+}$given by $\varphi^{+}\left(a+\lambda \cdot 1_{A}\right)=\varphi(a)+\lambda \cdot 1_{B}$. One immediately checks that the corresponding map $\varphi_{n}^{+}: M_{n}\left(A^{+}\right) \rightarrow M_{n}\left(B^{+}\right)$sends $\mathrm{GL}_{n}(A)$ into $\mathrm{GL}_{n}(B)$, and, using the description given in Remark 2.6, that it sends $\mathrm{TL}_{n}(A)$ into $\mathrm{TL}_{n}(B)$. Similarly, the map

$$
\left(x_{1}, \ldots, x_{n}\right) \mapsto\left(\varphi^{+}\left(x_{1}\right), \ldots, \varphi^{+}\left(x_{n}\right)\right)
$$

is easily seen to send $\operatorname{Lg}_{n}(A)$ into $\operatorname{Lg}_{n}(B)$ and to send $(0, \ldots, 0,1)$ to $(0, \ldots, 0,1)$. If $a \in \mathrm{GL}_{n}(A)$ and $x=\left(x_{1}, \ldots, x_{n}\right) \in \mathrm{Lg}_{n}(A)$, then the image of $a \cdot x$ in $\operatorname{Lg}_{n}(B)$ is equal to

$$
\varphi_{n}^{+}(a) \cdot\left(\varphi^{+}\left(x_{1}\right), \ldots, \varphi^{+}\left(x_{n}\right)\right) .
$$

It follows that $\operatorname{Lc}_{n}(A)$ is sent to $\operatorname{Lc}_{n}(B)$, and that we have a commutative diagram

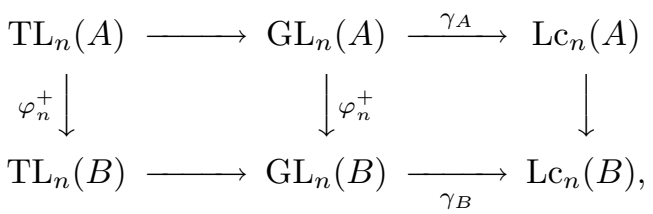

which is what naturality means.

The spaces $\mathrm{GL}_{n}(A)$ and $\mathrm{Lc}_{n}(A)$ are, in general, not connected. When we focus on homotopy theoretic properties of these spaces in subsequent sections, we will 
restrict our attention to the connected components $\mathrm{GL}_{n}(A)$ 。 and $\operatorname{Lc}_{n}(A)$ 。 of the basepoints. The following result justifies this restriction.

Proposition 2.9. Let $A$ be a Banach algebra. The connected components of the space $\mathrm{GL}_{n}(A)$ are path connected and all homeomorphic. The same holds for $\mathrm{Lc}_{n}(A)$. The homeomorphisms can be chosen to be natural with respect to both a single Banach algebra homomorphism and the map $\gamma$ of the fibration of Theorem 2.8(3).

Proof. The connected components of $\mathrm{GL}_{n}(A)$ and $\mathrm{Lc}_{n}(A)$ are open because these spaces are homeomorphic to open subsets of Banach spaces, by Theorem 2.8(5). Now note that connected open subsets of Banach spaces are path connected.

For the second part, let $a \in \mathrm{GL}_{n}(A)$. Then $b \mapsto a b$ is a homeomorphism from $\mathrm{GL}_{n}(A)$ to itself which sends $\mathrm{GL}_{n}(A)$ 。 to a connected, open, and closed subset of $\mathrm{GL}_{n}(A)$, which must be the connected component of $\mathrm{GL}_{n}(A)$ containing $a$. Similarly, the map $b e_{n} \mapsto a b e_{n}$, for $b \in \operatorname{GL}_{n}(A)$, is a homeomorphism from $\operatorname{Lc}_{n}(A)$ 。 to to the connected component of $\mathrm{Lc}_{n}(A)$ containing $a e_{n}$. By definition, every element of $\operatorname{Lc}_{n}(A)$ has the form $b e_{n}$ for some $b$, so this argument applies to all components.

For naturality, if $\varphi: A \rightarrow B$, and if $a \in \mathrm{GL}_{n}(A)$, we take the maps involving $B$ to be multiplication by $\varphi_{n}^{+}(a)$.

As a direct consequence of Theorem 2.8, we obtain a long exact homotopy sequence relating the homotopy groups of $\mathrm{GL}_{n}(A)$ and $\operatorname{Lc}_{n}(A)$. This sequence for $C^{*}$-algebras is due to Thomsen (Section 3 of 32 ). Note that, in the statement below, $\pi_{0}\left(\mathrm{GL}_{n-1}(A)\right)$ and $\pi_{0}\left(\mathrm{GL}_{n}(A)\right)$ are possibly non-abelian groups, and that $\pi_{0}\left(\operatorname{Lc}_{n}(A)\right)$ is just a set. That the sequence ends with 0 means that $\pi_{0}\left(\mathrm{GL}_{n}(A)\right) \rightarrow$ $\pi_{0}\left(\mathrm{Lc}_{n}(A)\right)$ is surjective.

Theorem 2.10. For a Banach algebra $A$ and for each $n>0$ there is a long exact sequence

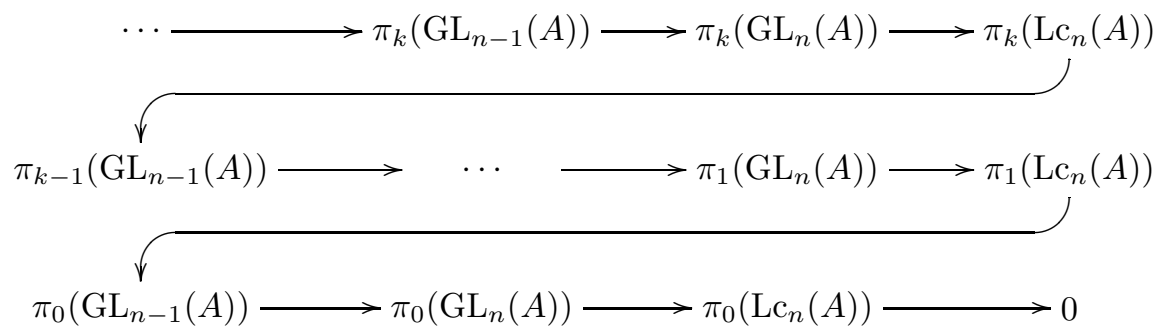

which is natural in $A$.

Proof. The long exact sequence in homotopy for the Serre fibration of Theorem $2.8(3)$ gives the sequence of the theorem with $\pi_{*}\left(\mathrm{TL}_{n}(A)\right)$ in place of $\pi_{*}\left(\mathrm{GL}_{n-1}(A)\right)$. (The map $\pi_{0}\left(\mathrm{GL}_{n}(A)\right) \rightarrow \pi_{0}\left(\mathrm{Lc}_{n}(A)\right)$ is surjective because $\mathrm{GL}_{n}(A) \rightarrow \mathrm{Lc}_{n}(A)$ is surjective.) The map $\mathrm{GL}_{n-1}(A) \rightarrow \mathrm{TL}_{n}(A)$ given by

$$
x \mapsto\left[\begin{array}{ll}
x & 0 \\
0 & 1
\end{array}\right]
$$

is a deformation retraction (see the description of $\mathrm{TL}_{n}(A)$ given in Remark 2.6) and hence induces an isomorphism on homotopy. This gives the sequence as stated.

Naturality is immediate from naturality of the fibration, Theorem 2.8(4). 
We now turn to the simplifications in the unital case. Stated informally, if $A$ is unital, then $\mathrm{GL}_{n}(A)$ is the invertible group of $M_{n}(A)$, the space $\mathrm{Lc}_{n}(A)$ is the set of last columns of invertible elements of $M_{n}(A)$, etc. The proof is immediate, and is omitted.

Proposition 2.11. Let $A$ be a unital Banach algebra, and identify $A^{+}$with the Banach algebra direct sum $A \oplus \mathbb{C}$ as in Remark 2.2. In the obvious way, further identify $M_{n}\left(A^{+}\right)$with $M_{n}(A) \oplus M_{n}(\mathbb{C})$ and $\left(A^{+}\right)^{n}$ with $A^{n} \oplus \mathbb{C}^{n}$. Then:

(1) The map $a \mapsto(a, 1)$ defines an isomorphism of topological groups from the group of invertible elements of $M_{n}(A)$ to $\mathrm{GL}_{n}(A)$.

(2) The map $x \mapsto(x,(0, \ldots, 0,1))$ defines a homeomorphism from

$$
\begin{aligned}
& \left\{\left(x_{1}, \ldots, x_{n}\right) \in A^{n}: \text { There are } y_{1}, \ldots, y_{n} \in A \text { such that } \sum_{k=1}^{n} y_{k} x_{k}=1\right\} \\
& \quad \text { to } \operatorname{Lg}_{n}(A) .
\end{aligned}
$$

These maps convert the obvious action of the invertible group of $M_{n}(A)$ on $\operatorname{Lg}_{n}(A)$ into the restriction of the action defined in Remark 2.4 to $\mathrm{GL}_{n}(A)$. In particular, they identify $\mathrm{Lc}_{n}(A)$ with the orbit of $(0, \ldots, 0,1)$ under the action of the invertible group of $M_{n}(A)$, and $\mathrm{TL}_{n}(A)$ with the invertible elements of $M_{n}(A)$ whose last column is $(0, \ldots, 0,1)$.

These maps are natural for continuous unital homomorphisms of unital Banach algebras.

\section{Commutative Banach algebras}

Suppose now that $A$ is a commutative unital Banach algebra. In this case, $\operatorname{Max}(A)$ is a non-empty compact Hausdorff space, and the Gelfand transform provides an algebra homomorphism

$$
\Gamma: A \rightarrow C(\operatorname{Max}(A)) .
$$

See Section 17 of 4 ] or Section 1.3 of 22]. Recall that if $A=C(X)$, then $\operatorname{Max}(A)=$ $X$, and the Gelfand transform is an isomorphism (Theorem 2.1.10 of [22]). In general, $\Gamma$ induces a continuous unital homomorphism

$$
\Gamma_{n}: A \otimes M_{n}(\mathbb{C}) \longrightarrow M_{n}(C(\operatorname{Max}(A)))=F\left(\operatorname{Max}(A), M_{n}(\mathbb{C})\right)
$$

which restricts to a natural continuous homomorphism

$$
\Gamma_{n}: \mathrm{GL}_{n}(A) \longrightarrow \mathrm{GL}_{n}(C(\operatorname{Max}(A)))=F\left(\operatorname{Max}(A), \mathrm{GL}_{n}(\mathbb{C})\right) .
$$

Here we recall that the group operation in the space $F\left(\operatorname{Max}(A), \mathrm{GL}_{n}(\mathbb{C})\right)$ is a pointwise multiplication of functions.

Remark 3.1. For naturality statements, it is important to note that a unital homomorphism $\varphi: A \rightarrow B$ of commutative unital Banach algebras induces, in a functorial manner, a continuous function $\varphi^{*}: \operatorname{Max}(B) \rightarrow \operatorname{Max}(A)$. If $M \subset B$ is a maximal ideal, then $\varphi^{*}(M)=\varphi^{-1}(M)$. If we think of the maximal ideal space as consisting of all (continuous) unital homomorphisms to $\mathbb{C}$, then $\varphi^{*}(\omega)=\omega \circ \varphi$.

It is then immediate that $A \mapsto C(\operatorname{Max}(A))$ is a covariant functor. Thus it makes sense to say that $\Gamma: A \rightarrow C(\operatorname{Max}(A))$ is natural. Similarly, $A \mapsto \check{H}^{*}(\operatorname{Max}(A) ; \mathbb{Q})$ is a covariant functor.

The following important result of Davie bridges the Gelfand transform gap. 
Theorem 3.2 (A. Davie). Let $A$ be a unital commutative Banach algebra with maximal ideal space $\operatorname{Max}(A)$. Then the natural homomorphism

$$
\Gamma_{n}: \mathrm{GL}_{n}(A) \longrightarrow F\left(\operatorname{Max}(A), \mathrm{GL}_{n}(\mathbb{C})\right)
$$

induced by the Gelfand transform is a homotopy equivalence.

Proof. This is a special case of Theorem 4.10 of [10].

We note in passing that Davie's work together with very elementary reasoning will substitute for the Shilov Idempotent Theorem and for the Arens-Royden Theorem in the computation of $H^{0}(\operatorname{Max}(A) ; \mathbb{Z})$ and $H^{1}(\operatorname{Max}(A) ; \mathbb{Z})$, respectively. For our purposes, the significance of this result is that it identifies $\operatorname{GL}_{n}(A)$, up to H-equivalence (as in Definition 4.2), as a particular gauge group. We devote the remainder of this section to obtaining a related identification of $\operatorname{Lc}_{n}(A)_{\circ}$.

Theorem 3.3. Let $A$ be a unital commutative Banach algebra with maximal ideal space $\operatorname{Max}(A)$. Then the Gelfand transform induces a natural map

$$
L \Gamma_{n}: \operatorname{Lc}_{n}(A) \text { 。 } \operatorname{Lc}_{n}(C(\operatorname{Max}(A)) \text { 。 }
$$

which is a homotopy equivalence.

Proof. Set $X=\operatorname{Max}(A)$. Apply Theorem 2.10 to $A$ and $C(X)$, and naturality in Theorem 2.10 to the Gelfand transform, obtaining a commutative diagram with long exact rows which ends with

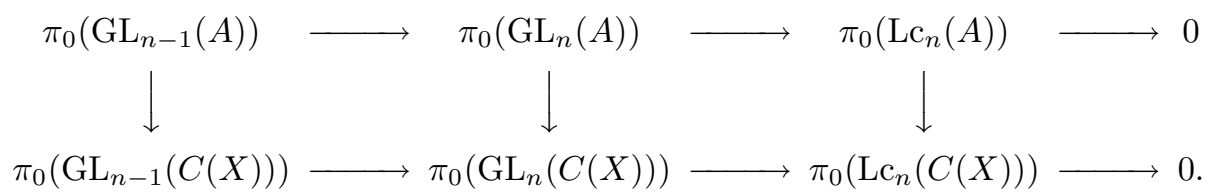

Theorem 3.2 and the Five Lemma imply that $\pi_{k}\left(\operatorname{Lc}_{n}(A)\right) \rightarrow \pi_{k}\left(\operatorname{Lc}_{n}(C(X))\right)$ is an isomorphism for all $k \geq 1$, and a direct argument shows that $\pi_{0}\left(\operatorname{Lc}_{n}(A)\right) \rightarrow$ $\pi_{0}\left(\operatorname{Lc}_{n}(C(X))\right)$ is a bijection of sets. Thus, $L \Gamma_{n}$ is a weak homotopy equivalence (on every connected component, by Proposition 2.91). Since these spaces are homotopy equivalent to CW complexes (by Theorem 2.8(5)), it follows from Theorem V.3.5 of 33] that $L \Gamma_{n}$ is a homotopy equivalence.

We now want to explicitly identify $\operatorname{Lc}_{n}(C(X))$.

Lemma 3.4. Let $X$ be a compact Hausdorff space. Identify $\operatorname{Lg}_{n}(C(X))$ with a subset of $C(X)^{n}$ as in Proposition 2.11(2), and further identify $C(X)^{n}$ with $F\left(X, \mathbb{C}^{n}\right)$ by sending $\left(f_{1}, \ldots, f_{n}\right)$ to $x \mapsto\left(f_{1}(x), \ldots, f_{n}(x)\right)$. Then the image of $\operatorname{Lg}_{n}(C(X))$ is exactly $F\left(X, \mathbb{C}^{n}-\{0\}\right)$.

Proof. Let $f \in F\left(X, \mathbb{C}^{n}\right)$, and write $f(x)=\left(f_{1}(x), \ldots, f_{n}(x)\right)$.

Suppose $f \in F\left(X, \mathbb{C}^{n}-\{0\}\right)$. Set

$$
g_{k}(x)=\frac{\overline{f_{k}(x)}}{\left|f_{1}(x)\right|^{2}+\cdots+\left|f_{n}(x)\right|^{2}} .
$$

(The denominator is never zero by hypothesis.) Then $g_{1} f_{1}+\cdots+g_{n} f_{n}=1$, so $\left(f_{1}, \ldots, f_{n}\right) \in \operatorname{Lg}_{n}(C(X))$.

Now suppose that there is $x \in X$ such that $f(x)=0$. Then everything in the ideal generated by $f_{1}, \ldots, f_{n}$ must vanish at $x$, so $\left(f_{1}, \ldots, f_{n}\right)$ is not in $\operatorname{Lg}_{n}(C(X))$. 
Corollary 3.5. Under the obvious map $F\left(X, \mathbb{C}^{n}\right) \rightarrow C(X)^{n}$, the image of the space $F\left(X, \mathbb{C}^{n}-0\right)$ 。 is exactly $\mathrm{Lc}_{n}(C(X))_{\circ}$.

Proof. Combine Lemma 3.4 and Theorem 2.8(6).

The following theorem gives the desired identification of the space $\operatorname{Lc}_{n}(A)$.

Theorem 3.6. Let $A$ be a commutative unital Banach algebra. Then there is a natural homotopy equivalence

$$
\operatorname{Lc}_{n}(A)_{\circ} \simeq F\left(\operatorname{Max}(A), S^{2 n-1}\right)_{\circ} .
$$

Proof. The Gelfand transform induces a natural homotopy equivalence

$$
\operatorname{Lc}_{n}(A) \stackrel{\simeq}{\longrightarrow} \operatorname{Lc}_{n}(C(\operatorname{Max}(A))) \text { 。 }
$$

by Theorem 3.3 Corollary 3.5 produces a natural homeomorphism

$$
\operatorname{Lc}_{n}(C(\operatorname{Max}(A)))_{\circ} \approx F\left(\operatorname{Max}(A), \mathbb{C}^{n}-\{0\}\right)_{\circ} .
$$

The deformation retraction

$$
\mathbb{C}^{n}-\{0\} \longrightarrow S^{2 n-1}
$$

given by $r(v)=v /\|v\|$ induces a natural homotopy equivalence

$$
F\left(\operatorname{Max}(A), \mathbb{C}^{n}-\{0\}\right)_{\circ} \stackrel{\simeq}{\longrightarrow} F\left(\operatorname{Max}(A), S^{2 n-1}\right)_{\circ},
$$

and so the composite

$$
\left(\operatorname{Lc}_{n}(A)\right)_{\circ} \rightarrow \operatorname{Lc}_{n}(C(\operatorname{Max}(A)))_{\circ} \rightarrow F\left(\operatorname{Max}(A), \mathbb{C}^{n}-\{0\}\right)_{\circ} \rightarrow F\left(\operatorname{Max}(A), S^{2 n-1}\right)_{\circ}
$$

is the required natural homotopy equivalence.

\section{H-Spaces, function spaces, and Rational homotopy}

Davie's Theorem (Theorem 3.2) gives a multiplicative homotopy equivalence between $\mathrm{GL}_{n}(A)$ and the function space $F\left(\operatorname{Max}(A), \mathrm{GL}_{n}(\mathbb{C})\right)$. Further, as mentioned in the Introduction, polar decomposition gives a multiplicative homotopy equivalence $U_{n}(\mathbb{C}) \stackrel{\simeq}{\longrightarrow} \mathrm{GL}_{n}(\mathbb{C})$. In this section, we give a complete description of the rationalization of the group $F(X, G)$ for $G$ a compact Lie group, provided $X$ is a finite complex. In fact, as is necessary for our analysis, we work in somewhat greater generality, allowing $G$ to be an $\mathrm{H}$-space with various restrictions. We also analyze the rationalization of the basepoint-preserving function spaces $F_{\bullet}(X, G)$. Throughout this section, we thus assume all spaces come equipped with a fixed, non-degenerate basepoint.

We let $G$ denote a connected H-space (Section III.4 of [33]). That is, we assume $G$ comes equipped with a continuous map $\mu: G \times G \rightarrow G$, for which there is an element $e \in G$ such that the restrictions of $\mu$ to $\{e\} \times G \rightarrow G$ and to $G \times\{e\} \rightarrow G$ are both homotopic to the identity map of $G$. The map $\mu$ is called the multiplication, and we will assume - as for topological groups - that the identity element $e$ is chosen as the basepoint of $G$. In what follows, we will write either the pair $(G, \mu)$ or just $G$, depending on whether or not we are concerned with the particular multiplication for $G$ or just the fact that $G$ is an $\mathrm{H}$-space.

While we mainly have in mind the case in which $(G, \mu)$ is a topological group, our results in this section require only that $\mu$ be homotopy-associative and have a homotopy inverse: a map $\nu: G \rightarrow G$ such that $\mu \circ(\nu \times 1) \circ \Delta: G \rightarrow G$ and $\mu \circ(1 \times \nu) \circ \Delta: G \rightarrow G$ are both null-homotopic. That is, we will require that $(G, \mu)$ 
be group-like as defined before Theorem III.4.14 of 33. We begin by recalling some basic facts about group-like spaces in ordinary homotopy theory.

We will often require that $G$ be a $\mathrm{CW}$ complex. In this context, recall the following result of I. James, Corollaries 1.2 and 1.3 of [16].

Proposition 4.1 (I. James). Let $(G, \mu)$ be a homotopy-associative H-space. For any $C W$ complex $X$ the set $[X, G]$ has the structure of a group. If $G$ is a $C W$ complex, then $(G, \mu)$ is group-like.

Definition 4.2. If $(G, \mu)$ and $\left(G^{\prime}, \mu^{\prime}\right)$ are H-spaces, then we say a map $F: G \rightarrow G^{\prime}$ is an $H$-map if it satisfies

$$
\mu^{\prime} \circ(F \times F) \sim F \circ \mu: G \times G \rightarrow G^{\prime},
$$

where " $\sim$ " denotes homotopy of maps. An H-map $F: G \rightarrow G^{\prime}$ that is a (weak) homotopy equivalence is called a (weak) $H$-equivalence. We say two H-spaces $G$ and $G^{\prime}$ are $H$-equivalent if there exists an H-equivalence $F: G \stackrel{\simeq}{\longrightarrow} G^{\prime}$.

Next we recall the definition of the homotopy nilpotency of a group-like space as introduced by Berstein and Ganea [3]. Suppose $(G, \mu)$ is a group-like space. Let

$$
\varphi_{2}: G \times G \rightarrow G
$$

denote the commutator map

$$
\varphi_{2}\left(g_{1}, g_{2}\right)=\mu\left(\mu\left(g_{1}, g_{2}\right), \mu\left(\nu\left(g_{1}\right), \nu\left(g_{2}\right)\right)\right) .
$$

Extend this definition by letting $\varphi_{1}: G \rightarrow G$ be the identity map and, for $n>1$, defining $\varphi_{n}: G^{n} \rightarrow G$ by the rule

$$
\varphi_{n}=\varphi_{2} \circ\left(\varphi_{n-1} \times \varphi_{1}\right) .
$$

The following corresponds to Definition 1.7 in [3].

Definition 4.3. Given a group-like space $(G, \mu)$, the homotopical nilpotency $\operatorname{Hnil}(G)$ of $(G, \mu)$ is the least integer $n$ such that the map $\varphi_{n+1}$ above is nullhomotopic. If $\operatorname{Hnil}(G)=1$ we say $(G, \mu)$ is homotopy-abelian.

Finally, recall that the homotopy groups of a homotopy-associative H-space $(G, \mu)$ come equipped with the Samelson bracket. (See Section X.5 of [33.) This is a bilinear pairing

$$
\langle,\rangle: \pi_{p}(G) \times \pi_{q}(G) \rightarrow \pi_{p+q}(G) .
$$

The following result is essentially Theorem 4.6 of $[3$.

Proposition 4.4 (I. Berstein and T. Ganea). If $(G, \mu)$ is H-equivalent to a loop space, then $\operatorname{Hnil}(G)$ is an upper bound for the longest non-vanishing Samelson bracket in $\pi_{*}(G)$.

Proof. By Theorem 4.6 of [3], $\operatorname{Hnil}(\Omega X)$ is an upper bound for the length of the longest non-vanishing Whitehead product in $\pi_{*}(X)$. Equivalently, by Theorem X.7.10 of [33], Hnil $(\Omega X)$ is an upper bound for the length of the longest nonvanishing Samelson bracket in $\pi_{*}(\Omega X)$.

Example 4.5. Recall that an Eilenberg-Mac Lane space $K(\pi, n)$ is a CW complex with only one non-zero homotopy group, namely $\pi_{n}(K(\pi, n))=\pi$. (See Section V.7 of 33 for the basic theory of Eilenberg-Mac Lane spaces.) If $\pi$ is abelian and $n \geq 1$, then $K(\pi, n)$ is homotopy equivalent to the loop space $\Omega K(\pi, n+1)$. Thus 
the Eilenberg-Mac Lane spaces are H-spaces with multiplication given by the usual product of loops. By Theorem V.7.13 of [33] this H-structure is unique up to homotopy. If $\pi$ is a countable abelian group, then $K(\pi, n)$ is actually an abelian topological group by the Corollary to Theorem 3 on page 360 of 20] (see also Milgram [19]). In particular, $\operatorname{Hnil}(K(\pi, n))=1$.

Example 4.6. More generally, let $\pi_{1}, \pi_{2}, \ldots$ be any sequence of countable abelian groups. Then the product space $\prod_{j \geq 1} K\left(\pi_{j}, j\right)$ also has the structure of an abelian topological group via the product multiplication. We refer to this multiplication as the standard multiplication for a product of Eilenberg-Mac Lane spaces. We emphasize that a product of Eilenberg-Mac Lane spaces may admit many non-Hequivalent H-structures. (For example, see Proposition Ia in [9.)

We now turn to the function space $F(X, G)$. If $(G, \mu)$ is an $\mathrm{H}$-space (respectively, a topological group), then $F(X, G)$ inherits an H-space structure (respectively, topological group structure) from that of $G$. Specifically, let

$$
\widehat{\mu}: F(X, G) \times F(X, G) \longrightarrow F(X, G)
$$

be given by

$$
\widehat{\mu}\left(f_{1}, f_{2}\right)(x)=\mu\left(f_{1}(x), f_{2}(x)\right)
$$

for $x \in X$ and $f_{1}, f_{2}: X \rightarrow G$. If the maps $f_{1}$ and $f_{2}$ preserve basepoints, then so does $\widehat{\mu}\left(f_{1}, f_{2}\right)$. Hence $F_{\bullet}(X, G)$ inherits an H-space structure in the same way. We recall that, when $X$ is the base space of a principal $G$-bundle, the group $F(X, G)$ is related to the gauge group of the bundle and has been studied extensively. (See, for example, 2. 2.) We will assume from now on that the function spaces $F(X, G)$ and $F_{\bullet}(X, G)$ come equipped with the multiplication induced by $G$. We next collect some basic facts regarding these $\mathrm{H}$-spaces.

Proposition 4.7. Let $X$ be a space and let $G$ be a homotopy-associative H-space. If either $X$ or $G$ is a $C W$ complex, then the path components of $F(X, G)$ are naturally homotopy equivalent to each other, and similarly for $F_{\bullet}(X, G)$.

Proof. In either case, Proposition 4.1 implies that the set $[X, G]$ of based homotopy classes is a group. This result is an extension to H-spaces (or to group-like spaces, as appropriate) of the argument of Proposition 2.9.

Proposition 4.8. Let $X$ be a compact space and let $G$ be a homotopy-associative $C W H$-space. Then $F(X, G)$ and $F_{\bullet}(X, G)$ are $H$-equivalent to group-like $C W$ complexes.

Proof. By Theorem 3 of [21, the function spaces have the homotopy type of $\mathrm{CW}$ complexes. The result follows from Proposition 4.1 .

Proposition 4.9. Let $X$ be a space and let $G$ be a path connected H-space. Then there is a natural weak H-equivalence

$$
F(X, G)_{\circ} \simeq_{w} G \times F_{\bullet}(X, G)_{\circ},
$$

where the right-hand side has the obvious product $H$-structure. If $X$ is a compact metric space and $G$ is a connected $C W$ complex, this is an H-equivalence that is natural in $X$. 
Proof. By assumption the basepoint $x_{0} \in X$ is non-degenerate, so that the inclusion $\left\{x_{0}\right\} \rightarrow X$ is a cofibration. This implies that the induced map

$$
F(X, Y) \rightarrow F\left(x_{0}, Y\right)
$$

is a fibration for any space $Y$. (See Theorem I.7.8 of [33.) This map is just the evaluation map $\omega: F(X, Y) \rightarrow Y$ defined by $\omega(f)=f\left(x_{0}\right)$. Taking $Y=G$ and restricting, we have a fibre sequence of path connected spaces

$$
F_{\bullet}(X, G)_{\circ} \stackrel{i}{\longrightarrow} F(X, G) \stackrel{\omega}{\longrightarrow} G .
$$

This fibration has a section $s$, which assigns to $g \in G$ the constant function $f(x)=g$. Therefore the long exact homotopy sequence breaks into split short exact sequences

$$
0 \longrightarrow \pi_{n}\left(F_{\bullet}(X, G)_{\circ}\right) \stackrel{i_{*}}{\longrightarrow} \pi_{n}\left(F(X, G) \stackrel{\omega_{*}}{\longrightarrow} \pi_{n}(G) \longrightarrow 0\right.
$$

for each $n$. Define an H-map $\varphi: G \times F_{\bullet}(X, G) \circ \rightarrow F(X, G)$ 。 by $\varphi=\widehat{\mu} \circ(s \times i)$, where $\widehat{\mu}$ is the multiplication on $F(X, G)$ 。 described before Proposition 4.7. It is straightforward to check that $\varphi$ induces an isomorphism on homotopy groups. Finally, if $X$ and $G$ satisfy the further hypotheses above, then, by Proposition 4.8 . the function spaces in question have the homotopy type of CW complexes. So a weak homotopy equivalence is a homotopy equivalence, by Theorem V.3.5 of [33].

Regarding the homotopical nilpotency of $F(X, G)_{\circ}$, we have the following result.

Theorem 4.10. Let $X$ be a space and $G$ a connected topological group, or, alternately, let $X$ be a compact space and $G$ a connected homotopy-associative $C W H$ space. Then

$$
\operatorname{Hnil}\left(F(X, G)_{\circ}\right)=\operatorname{Hnil}(G)
$$

Proof. With either hypothesis, $F(X, G)$ 。 is group-like, and so its homotopical nilpotency is well-defined. (In the second case, see Proposition 4.8.) As in the proof of Proposition 4.9, we have a section $s: G \rightarrow F(X, G)$ of the evaluation fibration $\omega: F(X, G)_{\circ} \rightarrow G$. It is easy to see that both $\omega$ and $s$ are H-maps - recall that the multiplication on $F(X, G)$ 。 is induced from that of $G$. Let

$$
\widehat{\varphi}_{n}: F(X, G)_{\circ}^{n} \rightarrow F(X, G)_{\circ}
$$

denote the commutator map for $F(X, G)_{\circ}$. Then we have

$$
\widehat{\varphi}_{n} \circ s^{n}=s \circ \varphi_{n}: G^{n} \rightarrow F(X, G)_{\circ} .
$$

The inequality $\operatorname{Hnil}\left(F(X, G)_{\circ}\right) \geq \operatorname{Hnil}(G)$ follows. On the other hand, suppose $\operatorname{Hnil}(G)=n$ so that $\varphi_{n+1}: G^{n+1} \rightarrow G$ is null-homotopic. We can identify

$$
F(X, G)_{\circ}^{n+1} \cong F\left(X, G^{n+1}\right)_{\circ} .
$$

The map $\widehat{\varphi}_{n+1}$ is then adjoint, via the exponential law, to the composition

$$
X \times F\left(X, G^{n+1}\right)_{\circ} \stackrel{\varepsilon}{\longrightarrow} G^{n+1} \stackrel{\varphi_{n+1}}{\longrightarrow} G
$$

where $\varepsilon(x, f)=f(x)$ is the generalized evaluation map. Since this composition is null-homotopic, $\widehat{\varphi}_{n+1}$ is null-homotopic. 
We now consider the H-space $F(X, G)$ 。 in the context of rational homotopy theory. To do so, however, we will need to make some further restrictions on $X$ and $G$. In particular, for the remainder of this section, all spaces (except function spaces) will be taken to be $\mathrm{CW}$ complexes.

We recall the basic facts about the rationalization of groups and spaces as developed in [15. A connected CW complex $X$ is nilpotent (Definition II.2.1 of [15]) if $\pi_{1}(X)$ is a nilpotent group and $\pi_{1}(X)$ acts nilpotently (Section I.4 of [15]) on each $\pi_{j}(X)$ for $j \geq 2$. A nilpotent space $X$ is a rational space if its homotopy groups $\pi_{j}(X)$ are $\mathbb{Q}$-vector spaces for each $j \geq 1$. (This is the case $P=\varnothing$ of Definition II.3.1 of 15.) If $\pi$ is a nilpotent group, then we denote by $\pi_{\mathbb{Q}}$ its rationalization in the sense of Definition I.1.1 of [15] (that is, the case $P=\varnothing$ there). If $\pi$ is abelian, such as for $\pi=\pi_{j}(X)$ with $j \geq 2$ and also for $\pi_{1}$ of an H-space, then $\pi_{\mathbb{Q}}$ is just $\pi \otimes \mathbb{Q}$. If $X$ is a nilpotent CW complex, then a function $f: X \rightarrow Y$ is a rationalization if $Y$ is rational and if $f$ induces an isomorphism

$$
f_{\sharp}: \pi_{j}(X)_{\mathbb{Q}} \stackrel{\cong}{\longrightarrow} \pi_{j}(Y)
$$

for each $j \geq 1$. This is not the same statement as Definition II.3.1 of [15], but is equivalent by Theorem 3B in Section II.3 of [15].

With this terminology, we have the following theorem.

Theorem 4.11 (Hilton, Mislin, and Roitberg). Every nilpotent $C W$ complex $X$ has a rationalization $e: X \rightarrow X_{\mathbb{Q}}$ where $X_{\mathbb{Q}}$ is a $C W$ complex. The space $X_{\mathbb{Q}}$ is unique up to homotopy equivalence.

Proof. Existence is Theorem 3B in Section II.3 of [15]. For uniqueness, suppose $f: X \rightarrow Y$ is some other rationalization. Following Definition II.3.1 of [15], $f$ induces a bijection

$$
f^{*}:\left[Y, X_{\mathbb{Q}}\right] \rightarrow\left[X, X_{\mathbb{Q}}\right]
$$

Thus there exists $h: Y \rightarrow X_{\mathbb{Q}}$ such that $h \circ f \simeq e$. Using Theorem 3B in Section II.3 of [15, we see that $h$ induces an isomorphism of rational homotopy groups since $f$ and $e$ do. Since $Y$ and $X_{\mathbb{Q}}$ are rational spaces, $h$ is a weak equivalence. Finally, since $Y$ and $X_{\mathbb{Q}}$ are CW complexes, Theorem V.3.5 of [33] implies that $h$ is a homotopy equivalence.

We use the notation $X \simeq_{\mathbb{Q}} Y$ to mean that $X$ and $Y$ are rationally equivalent spaces, that is, that $X_{\mathbb{Q}}$ and $Y_{\mathbb{Q}}$ are homotopy equivalent. We give some examples of rationalization maps which will figure prominently below.

Example 4.12. Consider an odd-dimensional sphere $S^{2 n-1}$. The groups $\pi_{j}\left(S^{2 n-1}\right)$ are finite except in the single degree $j=2 n-1$. (See Theorem 9.7.7 of [27].) So the rationalization of $S^{2 n-1}$ is a map

$$
e: S^{2 n-1} \rightarrow K(\mathbb{Q}, 2 n-1)
$$

corresponding to a non-trivial class in $H^{2 n-1}\left(S^{2 n-1} ; \mathbb{Q}\right)$. Thus, we have

$$
S^{2 n-1} \simeq_{\mathbb{Q}} K(\mathbb{Z}, 2 n-1) \simeq_{\mathbb{Q}} K(\mathbb{Q}, 2 n-1),
$$

and so, by Example 4.5, an odd-dimensional sphere has the rational homotopy type of an abelian topological group. We note in passing that even-dimensional spheres are slightly more complicated, having two non-trivial rational homotopy groups.

There are many more spaces, like $S^{2 n-1}$, whose rationalizations are EilenbergMac Lane spaces or products of them. 
Definition 4.13. Let $X$ be a connected nilpotent CW complex. We say $X$ is a rational $H$-space if $X_{\mathbb{Q}}$ is an H-space.

The following lemma and its corollary are well-known. (See, for instance, [26].) We include the statements and proofs for completeness.

Lemma 4.14. Let $G$ be a connected $C W H$-space. Then $G$ is nilpotent, and the rationalization of $G$ may be written in the form

$$
e: G \rightarrow \prod_{j \geq 1} K\left(\pi_{j}(G) \otimes \mathbb{Q}, j\right) .
$$

In particular, the rational homotopy groups of $G$ correspond to a space of algebra generators of $H^{*}(G ; \mathbb{Q})$. This rationalization is natural in the sense that a map of $H$-spaces $f: G_{1} \rightarrow G_{2}$ gives rise to a homotopy-commutative diagram in which the map on Eilenberg-Mac Lane spaces is induced by $f_{\sharp} \otimes 1: \pi_{*}\left(G_{1}\right) \otimes \mathbb{Q} \rightarrow \pi_{*}\left(G_{2}\right) \otimes \mathbb{Q}$.

Proof. The fundamental group of $G$ is abelian and acts trivially, that is, $G$ is a simple space. (See Theorem 7.3.9, and the preceding discussion, in 27.) So $G$ is nilpotent. By a classical theorem of H. Hopf (Corollary III.8.12 of [33]), the rational cohomology algebra $H^{*}(G ; \mathbb{Q})$ is the tensor product of an exterior algebra on odd generators with a polynomial algebra on even generators, that is, $H^{*}(G ; \mathbb{Q})$ is a free commutative graded algebra. (See Example 6 in Section 3 of [13].) Write $n_{1}, n_{2}, \ldots$ for the degrees of these generators. We then obtain a map $e: G \rightarrow \prod_{j} K\left(\mathbb{Q}, n_{j}\right)$ such that, on rational cohomology, the image of the fundamental class of $K\left(\mathbb{Q}, n_{j}\right)$ is the corresponding generator of $H^{*}(G ; \mathbb{Q})$. Since $H^{*}(G ; \mathbb{Q})$ is free over $\mathbb{Q}$, the map $e$ induces an isomorphism on rational cohomology. By Theorem 3B in Section II.3 of [15] again, $e$ is a rational equivalence.

Thus we see that it is sufficient to determine the rational homotopy groups of an $\mathrm{H}$-space, or more generally of a rational $\mathrm{H}$-space, in order to identify its rational homotopy type.

Corollary 4.15. Let $X$ be a rational H-space (Definition 4.13). Then there exists a rational equivalence of the form

$$
X \simeq_{\mathbb{Q}} \prod_{j \geq 1} K\left(\pi_{j}(X) \otimes \mathbb{Q}, j\right),
$$

which is natural in the same sense as in Lemma 4.14.

Proof. Apply Lemma 4.14 to $X_{\mathbb{Q}}$, which is a CW complex by Theorem 4.11, and is still connected, using the fact that the rationalization of a rational space is a homotopy equivalence.

Example 4.16. We specialize Lemma 4.14 to the Lie group $U_{n}(\mathbb{C})$. The cohomology $H^{*}\left(U_{n}(\mathbb{C}) ; \mathbb{Q}\right)$ is the exterior algebra on one generator in each odd degree from 1 through $2 n-1$. (See page 412 of [5.) Thus, using Example 4.12 ,

$$
U_{n}(\mathbb{C}) \simeq_{\mathbb{Q}} S^{1} \times S^{3} \times \cdots \times S^{2 n-1} \simeq_{\mathbb{Q}} \prod_{j=1}^{n} K(\mathbb{Q}, 2 j-1),
$$

as mentioned in the Introduction. Alternately, we may compute the rational homotopy of $U_{n}(\mathbb{C})$ from the long exact sequences of the fibrations $U_{k-1}(\mathbb{C}) \longrightarrow$ $U_{k}(\mathbb{C}) \longrightarrow S^{2 k-1}$ for $2 \leq k \leq n$ (used in the analysis in Section IV.10 of [33]). In 
particular, by IV.10.17 of 33, the map $U_{n-1}(\mathbb{C}) \rightarrow U_{n}(\mathbb{C})$ is an isomorphism on $\pi_{k}$ for $k \leq 2 n-3$.

Remark 4.17. The preceding examples display various possible situations which can occur in the context of $\mathrm{H}$-spaces and rationalization. As we show below (Corollaries 4.26 and 4.27), the rational equivalence $U_{n}(\mathbb{C}) \simeq_{\mathbb{Q}} \prod_{j=1}^{n} K(\mathbb{Q}, 2 j-1)$ can be taken to be multiplicative when the product of Eilenberg-Mac Lane spaces has the standard multiplication. On the other hand, for a general $\mathrm{H}$-space $G$, the map $G \rightarrow \prod_{j \geq 1} K\left(\pi_{j}(G) \otimes \mathbb{Q}, j\right)$ of Lemma 4.14 will rarely be multiplicative or even an $H$-map, even up to rational equivalence, if the product of Eilenberg-Mac Lane spaces has the standard multiplication. (See Example 4.22) Finally, the space $S^{2 n-1}$ is not an H-space for $n \neq 1,2,4$ (by J. F. Adams [1) and so the question of the multiplicativity of $S^{2 n-1} \rightarrow K(\mathbb{Q}, 2 n-1)$ in these cases is moot.

Turning to the rationalization of function spaces, the results we need depend on three basic developments. First, as has already been used, Theorem 3 of 21] shows that the path components of $F(X, Y)$ have the homotopy type of CW complexes provided $X$ is a compact metric space. (Recall we assume $Y$ has the homotopy type of a CW complex.) The second ingredient is Theorem II.3.11 of [15] and the discussion that follows it. Nilpotence of the spaces is Corollary II.2.6 of [15].

Theorem 4.18 (Hilton, Mislin, and Roitberg). Suppose that $X$ is a finite $C W$ complex and $Y$ is a nilpotent space with rationalization $e: Y \rightarrow Y_{\mathbb{Q}}$. Then the components of $F(X, Y)$ and $F_{\bullet}(X, Y)$ are all nilpotent spaces, and the induced maps

$$
e_{*}: F_{\bullet}(X, Y)_{\circ} \longrightarrow F_{\bullet}\left(X, Y_{\mathbb{Q}}\right) \circ \text { and } e_{*}: F(X, Y)_{\circ} \longrightarrow F\left(X, Y_{\mathbb{Q}}\right) \text { 。 }
$$

given by composition with e are rationalizations.

The third ingredient is an early result of R. Thom (Theorem 2 of [31]).

Theorem 4.19 (R. Thom). Let $X$ be a Hausdorff topological space. Then there is a natural isomorphism

$$
\pi_{j}\left(F(X, K(\pi, n))_{\circ}\right) \cong H^{n-j}(X ; \pi)
$$

for $j \geq 1$.

If $X$ is not a finite CW complex, then one must specify which cohomology theory is being used: Thom is using singular cohomology. This is not the best choice for compact spaces; Čech theory is better, and in general the natural map from Čech theory to singular theory is neither injective nor surjective. We use Thom's result only for $X$ a finite complex.

Combining the facts above, we obtain the following result.

Theorem 4.20. Let $X$ be a finite $C W$ complex and let $G$ be a connected $C W H$ space or, more generally, a rational $H$-space. Let $V_{j}$ and $\widetilde{V}_{j}$ be the rational vector spaces

$$
V_{j}=\bigoplus_{l \geq j} H^{l-j}\left(X ; \pi_{l}(G) \otimes \mathbb{Q}\right) \quad \text { and } \quad \widetilde{V}_{j}=\bigoplus_{l \geq j} \widetilde{H}^{l-j}\left(X ; \pi_{l}(G) \otimes \mathbb{Q}\right) .
$$

Then there are natural rational equivalences

$$
F(X, G)_{\circ} \simeq_{\mathbb{Q}} \prod_{j \geq 1} K\left(V_{j}, j\right) \quad \text { and } \quad F_{\bullet}(X, G)_{\circ} \simeq_{\mathbb{Q}} \prod_{j \geq 1} K\left(\widetilde{V}_{j}, j\right) .
$$


Proof. Since $G$ is a rational H-space, we apply Corollary 4.15 to write its rationalization as a product of Eilenberg-Mac Lane spaces

$$
G_{\mathbb{Q}} \simeq \prod_{l \geq 1} K\left(\pi_{l}(G) \otimes \mathbb{Q}, l\right) .
$$

By Theorem 4.18, we have rational equivalences

$$
F(X, G)_{\circ} \simeq_{\mathbb{Q}} F\left(X, G_{\mathbb{Q}}\right)_{\circ} \text { and } F_{\bullet}(X, G)_{\circ} \simeq_{\mathbb{Q}} F \cdot\left(X, G_{\mathbb{Q}}\right)_{\circ} .
$$

Now $F\left(X, G_{\mathbb{Q}}\right)$ 。 and $F_{\bullet}\left(X, G_{\mathbb{Q}}\right) \circ$ are H-spaces. Thus, by Lemma 4.14, to prove the theorem it suffices to compute the (rational) homotopy groups of these spaces.

Using the standard homeomorphism of function spaces $F\left(X, \prod_{l} Y_{l}\right) \approx \prod_{l} F\left(X, Y_{l}\right)$, we obtain

$$
F\left(X, G_{\mathbb{Q}}\right)_{\circ} \simeq F\left(X, \prod_{l \geq 1} K\left(\pi_{l}(G) \otimes \mathbb{Q}, l\right)\right)_{\circ} \approx \prod_{l \geq 1} F\left(X, K\left(\pi_{l}(G) \otimes \mathbb{Q}, l\right)\right)_{\circ} .
$$

Thom's result, Theorem 4.19, now gives

$$
\pi_{j}\left(F\left(X, K\left(\pi_{l}(G) \otimes \mathbb{Q}, l\right)\right)_{0}\right) \cong H^{l-j}\left(X ; \pi_{l}(G) \otimes \mathbb{Q}\right) .
$$

Thus we see $\pi_{j}\left(F\left(X, G_{\mathbb{Q}}\right)_{\circ}\right) \cong V_{j}$, as needed. Next, using this isomorphism and the split short exact sequence (4.1) we compute $\pi_{j}\left(F_{\bullet}\left(X, G_{\mathbb{Q}}\right)_{\circ}\right) \cong \widetilde{V}_{j}$, which completes the proof.

The ingredients of Theorem 4.20 have been combined before in the same way, for specific $X$ and $G$. (See, for instance, the computations in Section 2 of 22.) While Theorem 4.20 determines the full rational homotopy type of the function spaces of interest to us, these function spaces are $\mathrm{H}$-spaces, and so it is natural to ask whether we can determine their structure as such, at least up to rational H-equivalence. We take up this question for the remainder of the section.

Following the discussion before Theorem II.1.8 of [15], if $(G, \mu)$ is an H-space, then the rationalization $G_{\mathbb{Q}}$ admits a multiplication $\mu_{\mathbb{Q}}$ making the rationalization map $e: G \rightarrow G_{\mathbb{Q}}$ an H-map. Moreover, this $\mathrm{H}$-space structure on $G_{\mathbb{Q}}$ is uniquely determined by that of $G$ in the sense that if $\left(\widetilde{G}_{\mathbb{Q}}, \widetilde{\mu}_{\mathbb{Q}}\right)$ represents a second rationalization of $(G, \mu)$, then $\left(G_{\mathbb{Q}}, \mu_{\mathbb{Q}}\right)$ and $\left(\widetilde{G}_{\mathbb{Q}}, \widetilde{\mu}_{\mathbb{Q}}\right)$ are H-equivalent. It is easy to see that if $(G, \mu)$ is group-like, then so is $\left(G_{\mathbb{Q}}, \mu_{\mathbb{Q}}\right)$.

Definition 4.21. We say two CW H-spaces $(G, \mu)$ and $\left(G^{\prime}, \mu^{\prime}\right)$ are rationally $H$ equivalent if their rationalizations $\left(G_{\mathbb{Q}}, \mu_{\mathbb{Q}}\right)$ and $\left(G_{\mathbb{Q}}^{\prime}, \mu_{\mathbb{Q}}^{\prime}\right)$ are H-equivalent.

Example 4.22. The rationalization $K(\mathbb{Z}, n) \rightarrow K(\mathbb{Q}, n)$ is clearly a rational Hequivalence. On the other hand, the rationalization $\Omega S^{2} \rightarrow K(\mathbb{Q}, 1) \times K(\mathbb{Q}, 2)$ is not a rational $\mathrm{H}$-equivalence if both sides have the standard loop multiplications. To see that these spaces are not H-equivalent, we argue as follows. Let $\iota \in \pi_{2}\left(S^{2}\right)$ be the homotopy class of the identity map. As is well-known, $[\iota, \iota] \in \pi_{3}\left(S^{2}\right)$ is of infinite order (see Theorem XI.2.5 of [33]), yielding a non-zero Whitehead bracket in $\pi_{*}\left(S_{\mathbb{Q}}^{2}\right)$. Under the identification of Whitehead brackets in $\pi_{*}\left(S_{\mathbb{Q}}^{2}\right)$ with Samelson brackets in $\pi_{*}\left(\Omega S_{\mathbb{Q}}^{2}\right)$ (Theorem X.7.10 of [33]), there is a corresponding non-zero Samelson bracket in $\pi_{*}\left(\Omega S_{\mathbb{Q}}^{2}\right)$. By Proposition 4.4 we have $\operatorname{Hnil}\left(\Omega S_{\mathbb{Q}}^{2}\right) \geq 2$. On the other hand, $K(\mathbb{Q}, 1) \times K(\mathbb{Q}, 2)$ is homotopy-abelian. 
Definition 4.23. Given a connected CW homotopy-associative H-space $(G, \mu)$, the rational homotopical nilpotency $\operatorname{Hnil}_{\mathbb{Q}}(G)$ is the homotopical nilpotency of the rationalization $\left(G_{\mathbb{Q}}, \mu_{\mathbb{Q}}\right)$ of $(G, \mu)$, that is, $\operatorname{Hnil}_{\mathbb{Q}}(G)=\operatorname{Hnil}\left(G_{\mathbb{Q}}\right)$. We say $(G, \mu)$ is rationally homotopy-abelian if $\operatorname{Hnil}_{\mathbb{Q}}(G)=1$.

The Samelson bracket in $\pi_{*}(G)$ induces a bracket in the rationalization giving $\pi_{*}(G) \otimes \mathbb{Q}$ the structure of a graded Lie algebra as in the following definition. (See the beginning of Section 21 of [13].)

Definition 4.24. A positively graded vector space $L$ over $\mathbb{Q}$ is a graded Lie algebra if $L$ comes equipped with a bilinear, degree zero pairing $\langle$,$\rangle satisfying$

(1) Anti-symmetry: $\langle\alpha, \beta\rangle=-(-1)^{\operatorname{deg}(\alpha) \operatorname{deg}(\beta)}\langle\beta, \alpha\rangle$.

(2) Jacobi identity: $\langle\alpha,\langle\beta, \gamma\rangle\rangle=\langle\langle\alpha, \beta\rangle, \gamma\rangle+(-1)^{\operatorname{deg}(\alpha) \operatorname{deg}(\beta)}\langle\beta,\langle\alpha, \gamma\rangle\rangle$.

By Theorems X.5.1 and X.5.4 of [33], after tensoring with the rationals, $\pi_{*}(G) \otimes \mathbb{Q}$ with the induced bracket is a graded Lie algebra over $\mathbb{Q}$.

The following result can be deduced in various ways; our proof is based on results of H. Scheerer in [26].

Theorem 4.25. Let $(G, \mu)$ be a connected $C W$ homotopy-associative H-space. The following are equivalent:

(1) $(G, \mu)$ has the rational H-type of an abelian topological group.

(2) $(G, \mu)$ is rationally homotopy-abelian.

(3) The Samelson Lie algebra $\pi_{*}(G) \otimes \mathbb{Q}$, described above, is abelian, that is, all brackets are zero.

(4) The rational equivalence

$$
e: G \rightarrow \prod_{j \geq 1} K\left(\pi_{j}(G) \otimes \mathbb{Q}, j\right)
$$

of Lemma 4.14 is a rational H-equivalence, where the product of EilenbergMac Lane spaces has the standard multiplication.

Proof. The implication (1) $\Longrightarrow$ (2) is immediate from definitions. The implication (4) $\Longrightarrow$ (11) is the result of Milnor mentioned in Example 4.5 (Corollary to Theorem 3 of 20$]$ ).

We prove (2) $\Longrightarrow$ (3). Proposition 4.1 implies that $(G, \mu)$ is group-like, so $\left(G_{\mathbb{Q}}, \mu_{\mathbb{Q}}\right)$ is group-like, too. Corollary 2 in Section 0.1 of $[26$, therefore shows that $(G, \mu)$ is rationally $\mathrm{H}$-equivalent to a loop space $\Omega X$ with the usual multiplication of loops. Now use Proposition 4.4.

Finally, for (3) $\Longrightarrow$ (4) use Corollary 3 of [26], which gives a bijection between the set of H-maps between two rational group-like spaces and the space of homomorphisms between their rational Samelson algebras. The rational Samelson algebra of $\prod_{j \geq 1} K\left(\pi_{j}(G), j\right)$ is abelian because $\prod_{j \geq 1} K\left(\pi_{j}(G), j\right)$ is rationally abelian. So if $\pi_{*}(G) \otimes \mathbb{Q}$ is abelian, then $e$ trivially induces an isomorphism of rational Samelson algebras and is thus a rational $\mathrm{H}$-equivalence.

The following useful consequences are well-known.

Corollary 4.26. The standard loop multiplication on a product of Eilenberg Mac Lane spaces is the unique group-like, homotopy-abelian H-structure up to rational H-equivalence.

Proof. Use (2) $\Longleftrightarrow$ (4) in Theorem 4.25. 
Corollary 4.27. Let $G$ be a connected $C W$ homotopy-associative H-space. Suppose $H^{*}(G ; \mathbb{Q})$ is finite-dimensional. Then $G$ is rationally homotopy-abelian.

Proof. Referring to the proof of Lemma 4.14 we see that finite-dimensionality of $H^{*}(G ; \mathbb{Q})$ implies that $H^{*}(G ; \mathbb{Q})$ is an exterior algebra on odd-degree generators. By that same result, we conclude that $\pi_{*}(G) \otimes \mathbb{Q}$ is zero in even degrees. Thus, for degree reasons, the rational Samelson bracket on $\pi_{*}(G) \otimes \mathbb{Q}$ is trivial. This is Condition (3) of Theorem 4.25, so we conclude that $G$ is rationally homotopyabelian.

Finally, we identify the H-spaces $F(X, G)$ 。 and $F_{\bullet}(X, G) \circ$, for $X$ and $G$ finite complexes, up to rational H-equivalence. Note that a continuous map $f: X \rightarrow$ $Y$ induces H-maps $f^{*}: F(Y, G)_{\circ} \rightarrow F(X, G)_{\circ}$ and $f^{*}: F_{\bullet}(Y, G)_{\circ} \rightarrow F_{\bullet}(X, G)_{\circ}$. Similarly, an H-map $h: G \rightarrow H$ induces H-maps $h_{*}: F(X, G)_{\circ} \rightarrow F(X, H) \circ$ and $h_{*}: F_{\bullet}(X, G)_{\circ} \rightarrow F_{\bullet}(X, H)_{\circ}$.

Theorem 4.28. Let $X$ be a finite $C W$ complex. Let $G$ be a connected $C W$ homotopy-associative $H$-space with $H^{*}(G ; \mathbb{Q})$ finite-dimensional. Then $F(X, G)$ 。 and $F_{\bullet}(X, G) \circ$ are homotopy-abelian after rationalization. Consequently, the rational equivalences of Theorem 4.20 are actually rational H-equivalences where the products of Eilenberg-Mac Lane spaces have standard multiplication. Moreover, these rational $H$-equivalences are natural with respect to maps $f: X \rightarrow Y$ and $H$-maps $h: G \rightarrow H$.

Proof. Corollary 4.27 implies that $G$ is rationally homotopy-abelian. Thus, by Theorem 4.10, $F\left(X, G_{\mathbb{Q}}\right)$ 。 is rationally homotopy-abelian. Using Proposition 4.9 and the fact that the rational Samelson algebra of an H-product is the product of the Samelson algebras (see Example 21.4 of 13 ), we see that the rational Samelson algebra of $F_{\bullet}(X, G)$ 。 is also abelian. Thus $F_{\bullet}(X, G)$ 。 is rationally homotopy-abelian by Theorem 4.25. The naturality assertions now follow from the naturality of the rational homotopy equivalence of a rational $\mathrm{H}$-space with the appropriate product of Eilenberg-Mac Lane spaces as in Corollary 4.15.

\section{Conclusion: Passage to limits}

We now prove the main results of the paper. First, using our results above we give a preliminary version of Theorem 1 in the special case in which the maximal ideal space $\operatorname{Max}(A)$ of $A$ happens to be a finite complex.

Theorem 5.1. Suppose that $A$ is a commutative unital Banach algebra and $\operatorname{Max}(A)$ is a finite $C W$ complex. Let $V_{h, j}=H^{2 j-1-h}(\operatorname{Max}(A) ; \mathbb{Q})$. Then there is a rational H-equivalence

$$
\mathrm{GL}_{n}(A)_{\circ} \simeq_{\mathbb{Q}} \prod_{j=1}^{n} \prod_{h=1}^{2 j-1} K\left(V_{h, j}, h\right),
$$

where the product of Eilenberg-Mac Lane spaces has standard loop multiplication. The equivalence is natural with respect to homomorphisms between commutative unital Banach algebras with the maximal ideal space a finite complex. Moreover, for fixed $A$ with $\operatorname{Max}(A)$ a finite complex the equivalence is natural in $n$ in the following sense: For each $n>1$, let $i: \mathrm{GL}_{n-1}(A)_{\circ} \rightarrow \mathrm{GL}_{n}(A)$ 。 denote the inclusion in the 
upper left corner. Then, after the identifications

$$
\pi_{k}\left(\mathrm{GL}_{n-1}(A)_{\circ}\right) \otimes \mathbb{Q} \cong \bigoplus_{j=1}^{n-1} V_{k, j} \text { and } \pi_{k}\left(\mathrm{GL}_{n}(A)_{\circ}\right) \otimes \mathbb{Q} \cong \bigoplus_{j=1}^{n} V_{k, j}
$$

for each $k \geq 1$ given above, the map

$$
i_{\sharp} \otimes 1: \pi_{k}\left(\mathrm{GL}_{n-1}(A)_{\circ}\right) \otimes \mathbb{Q} \rightarrow \pi_{k}\left(\mathrm{GL}_{n}(A) \circ\right) \otimes \mathbb{Q}
$$

corresponds to the inclusion of vector spaces

$$
\bigoplus_{j=1}^{n-1} V_{k, j} \hookrightarrow \bigoplus_{j=1}^{n} V_{k, j}
$$

Proof. By Theorem 3.2 we have an H-equivalence

$$
\mathrm{GL}_{n}(A)_{\circ} \simeq F\left(\operatorname{Max}(A), \mathrm{GL}_{n}(\mathbb{C})\right)_{\circ} .
$$

The rational homotopy groups of $\mathrm{GL}_{n}(\mathbb{C}) \simeq U_{n}(\mathbb{C})$ occur in degrees $1,3, \ldots, 2 n-$ 1 by Example 4.16. Theorem 4.20 thus gives the needed rational equivalence. Since $H^{*}\left(\mathrm{GL}_{n}(\mathbb{C}), \mathbb{Q}\right)$ is finite-dimensional, Theorem 4.28 implies this equivalence is actually a rational H-equivalence. Naturality with respect to Banach algebra homomorphisms is a direct consequence of the naturality given in that theorem. For naturality with respect to $n$, we first need to know what the inclusion $\mathrm{GL}_{n-1}(\mathbb{C})$ 。 $\mathrm{GL}_{n}(\mathbb{C})$ 。 does on the rational homotopy groups. For this, use the retractions to the corresponding unitary groups and Example 4.16. Naturality with respect to $n$ now follows from the naturality in Theorem 4.28 and the fact that the inclusion $i: \mathrm{GL}_{n-1}(A)_{\circ} \rightarrow \mathrm{GL}_{n}(A)$ 。 corresponds, via the H-equivalence of Theorem 3.2 to the map $F\left(\operatorname{Max}(A), \mathrm{GL}_{n-1}(\mathbb{C})\right) \rightarrow F\left(\operatorname{Max}(A), \mathrm{GL}_{n}(\mathbb{C})\right)$ 。 induced by the inclusion $\mathrm{GL}_{n-1}(\mathbb{C})_{\circ} \hookrightarrow \mathrm{GL}_{n}(\mathbb{C})_{\circ}$.

We deduce the general case by considering limits of direct systems in our various settings. Recall that a unital homomorphism $\varphi: A \rightarrow B$ between unital Banach algebras induces a group homomorphism $\mathrm{GL}_{n}(\varphi): \mathrm{GL}_{n}(A) \rightarrow \mathrm{GL}_{n}(B)$. We first consider various direct systems in the $C^{*}$-algebra setting. The material here is in principle well known, but we have been unable to find a reference giving it in the generality needed here. The closest we are aware of is Section 4 of [14. Unfortunately, in this reference it is assumed throughout that all the maps in the direct system are injective, a condition which does not hold in our application.

Let $\Lambda$ be a directed set. Let $\left(A_{\lambda}\right)_{\lambda \in \Lambda}$ be a direct system of $C^{*}$-algebras, indexed by $\Lambda$ with $C^{*}$-algebra maps $\varphi_{\lambda, \mu}: A_{\lambda} \rightarrow A_{\mu}$. The direct limit $A=\lim _{\lambda} A_{\lambda}$ exists in the category of $C^{*}$-algebras. In fact, we have the following characterization.

Theorem 5.2. The direct limit $A=\lim _{\lambda} A_{\lambda}$ of a system of $C^{*}$-algebra maps $\varphi_{\lambda, \mu}: A_{\lambda} \rightarrow A_{\mu}$ is characterized by the existence of $C^{*}$-algebra homomorphisms $\varphi_{\lambda, \infty}: A_{\lambda} \rightarrow A$ such that:

(1) $\varphi_{\mu, \infty} \circ \varphi_{\lambda, \mu}=\varphi_{\lambda, \infty}$ whenever $\mu \geq \lambda$.

(2) If $a \in A_{\lambda}$ and $\varphi_{\lambda, \infty}(a)=0$, then $\lim _{\mu}\left\|\varphi_{\lambda, \mu}(a)\right\|=0$.

(3) $\bigcup_{\lambda \in \Lambda} \varphi_{\lambda, \infty}\left(A_{\lambda}\right)$ is dense in $A$.

Proof. See the proof of Proposition 2.5.1 of [23] for the construction, and see Theorem 6.1 .2 of [22] and the preceding discussion for the case $\Lambda=\mathbb{N}$. That these 
properties characterize the direct limit for $\Lambda=\mathbb{N}$ is implicit in the proof of Theorem 6.1.2(b) of [22], and the proof for a general index set is the same. (Murphy has a slightly different formulation for the second condition. The key fact in the proof is that an injective ${ }^{*}$-algebra homomorphism of $C^{*}$-algebras is isometric, even without assuming continuity to begin with.)

We emphasize that $\bigcup_{\lambda \in \Lambda} \varphi_{\lambda, \infty}\left(A_{\lambda}\right)$ is usually not the entire direct limit, merely a dense subalgebra.

Given a direct system $\left(A_{\lambda}\right)_{\lambda \in \Lambda}$ of $C^{*}$-algebras with maps $\varphi_{\lambda, \mu}: A_{\lambda} \rightarrow A_{\mu}$, we obtain direct systems of matrix and function spaces. Recall that $M_{n}(A)$ denotes the space of $n \times n$ matrices with entries in $A$. Write $\widetilde{\varphi}_{\lambda, \mu}: M_{n}\left(A_{\lambda}\right) \rightarrow M_{n}\left(A_{\mu}\right)$ for the map induced by applying $\varphi_{\lambda, \mu}$ entry-wise. For function spaces in the $C^{*}$-algebra context, we introduce the following notation. We deviate from our usual notation for function spaces to follow the standard notation $C(Y, A)$ for $C^{*}$-algebras.

Notation 5.3. For any $C^{*}$-algebra $A$ and any space $Y$ let $C(Y, A)$ denote the $C^{*}$ algebra of continuous functions $b: Y \rightarrow A$, with pointwise operations and the supremum norm $\|a\|=\sup _{y \in Y}\|a(y)\|$. If moreover $\varphi: A \rightarrow B$ is a homomorphism of $C^{*}$-algebras, we write $\bar{\varphi}: C(Y, A) \rightarrow C(Y, B)$ for the map obtained by composition with $\varphi$.

The following result shows that the direct limits in both cases are as expected. The result is a special case of the statement that direct limits commute with maximal completed tensor products, but our proof avoids the technicalities of completed tensor products.

Lemma 5.4. Let $\left(A_{\lambda}\right)_{\lambda \in \Lambda}$ be a direct system of $C^{*}$-algebras, with maps $\varphi_{\lambda, \mu}: A_{\lambda} \rightarrow$ $A_{\mu}$, and let $A=\lim _{\lambda} A_{\lambda}$. Let $Y$ be a compact Hausdorff space. Then:

(1) For $n \in \mathbb{N}$ there is a natural isomorphism $\lim _{\longrightarrow \lambda} M_{n}\left(A_{\lambda}\right) \cong M_{n}(A)$.

(2) Then there is a natural isomorphism $\lim _{\lambda} \vec{C}\left(Y, A_{\lambda}\right) \cong C(Y, A)$.

Proof. For (11), the maps are obtained by applying $\varphi_{\lambda, \infty}$ to each entry. In the characterization of the direct limit (Theorem 5.2), Parts (11) and (3) are immediate, and Part (2) follows from the fact that if the entries of a net of matrices converge to zero, then so do the matrices.

We prove (2). The maps $C\left(Y, A_{\lambda}\right) \rightarrow C\left(Y, A_{\mu}\right)$ and $C\left(Y, A_{\lambda}\right) \rightarrow C(Y, A)$ are of course $\bar{\varphi}_{\lambda, \mu}$ and $\bar{\varphi}_{\lambda, \infty}$, as in Notation 5.3 .

Part (11) of the characterization of the direct limit is immediate. For Part (2), let $b \in C\left(Y, A_{\lambda}\right)$ with $\bar{\varphi}_{\lambda, \infty}(b)(y)=0$. Because homomorphisms of $C^{*}$-algebras are norm decreasing, the functions $f_{\mu}(y)=\left\|\varphi_{\lambda, \mu}(b(y))\right\|$ decrease pointwise to 0 . By Dini's Theorem (Problem E in Chapter 7 of [17]), the convergence is uniform, that is, $\lim _{\mu}\left\|\bar{\varphi}_{\lambda, \mu}(b)\right\|=0$.

We finish by proving Part (3) of the characterization of the direct limit. Let $b \in C(Y, A)$, and let $\varepsilon>0$. Choose a finite partition of unity $\left(f_{1}, \ldots, f_{n}\right)$ such that whenever $x, y \in \operatorname{supp}\left(f_{k}\right)$, then $\|b(x)-b(y)\|<\frac{1}{3} \varepsilon$. Choose $y_{k} \in \operatorname{supp}\left(f_{k}\right)$. Choose $\lambda_{k} \in \Lambda$ and $c_{k} \in A_{\lambda_{k}}$ such that $\left\|\varphi_{\lambda_{k}, \infty}\left(c_{k}\right)-b\left(y_{k}\right)\right\|<\frac{1}{3} \varepsilon$. Choose $\lambda \in \Lambda$ such that $\lambda \geq \lambda_{k}$ for all $k$, and let $a_{k}=\varphi_{\lambda_{k}, \lambda}\left(c_{k}\right) \in A_{\lambda}$. Then $\left\|\varphi_{\lambda, \infty}\left(a_{k}\right)-b\left(y_{k}\right)\right\|<\frac{1}{3} \varepsilon$. Define $a \in A_{\lambda}$ by $a(y)=\sum_{k=1}^{n} f_{k}(y) a_{k}$. For $y \in Y$ we have $\left\|b\left(y_{k}\right)-b(y)\right\|<\frac{1}{3} \varepsilon$ 
whenever $f_{k}(y) \neq 0$, whence

$$
\begin{aligned}
\left\|\varphi_{\lambda, \infty}(a(y))-b(y)\right\| & \leq \sum_{k=1}^{n} f_{k}(y)\left[\left\|a_{k}-b\left(y_{k}\right)\right\|+\left\|b\left(y_{k}\right)-b(y)\right\|\right] \\
& \leq \sum_{k=1}^{n} 2\left(\frac{\varepsilon}{3}\right) f_{k}(y)=\frac{2 \varepsilon}{3} .
\end{aligned}
$$

So $\left\|\bar{\varphi}_{\lambda, \infty}(a)-b\right\|<\varepsilon$.

Now given a direct system $\left(A_{\lambda}\right)_{\lambda \in \Lambda}$ of unital $C^{*}$-algebras with unital maps $\varphi_{\lambda, \mu}: A_{\lambda} \rightarrow A_{\mu}$, we obtain a direct system $\left(\mathrm{GL}_{n}\left(A_{\lambda}\right)\right)_{\lambda \in \Lambda}$ of invertible groups with structure maps $\mathrm{GL}_{n}\left(\varphi_{\lambda, \mu}\right): \mathrm{GL}_{n}\left(A_{\lambda}\right) \rightarrow \mathrm{GL}_{n}\left(A_{\mu}\right)$. The following result is implicit in the usual direct proofs that $K_{1}$ commutes with direct limits of $C^{*}$-algebras. The proofs we know in the literature, however, instead use Bott periodicity and the result for $K_{0}$.

Theorem 5.5. Let $\left(A_{\lambda}\right)_{\lambda \in \Lambda}$ be a direct system of unital $C^{*}$-algebras, with unital maps $\varphi_{\lambda, \mu}: A_{\lambda} \rightarrow A_{\mu}$, and let $A=\lim _{\lambda} A_{\lambda}$. Then the maps

$$
\mathrm{GL}_{n}\left(\varphi_{\lambda, \infty}\right): \mathrm{GL}_{n}\left(A_{\lambda}\right) \rightarrow \mathrm{GL}_{n}(A)
$$

induce a natural isomorphism

$$
\underset{\lambda}{\underset{\lambda}{\lim }} \pi_{k}\left(\mathrm{GL}_{n}\left(A_{\lambda}\right)\right) \cong \pi_{k}\left(\mathrm{GL}_{n}(A)\right)
$$

for $k \geq 0$.

Proof. Following Notation 5.3, set $B_{\lambda}=C\left(S^{k}, M_{n}\left(A_{\lambda}\right)\right)$ and $B=C\left(S^{k}, M_{n}(A)\right)$. Then $\pi_{k}\left(\mathrm{GL}_{n}\left(A_{\lambda}\right)\right)=\pi_{0}\left(\mathrm{GL}_{1}\left(B_{\lambda}\right)\right)$ and $\pi_{k}\left(\mathrm{GL}_{n}(A)\right)=\pi_{0}\left(\mathrm{GL}_{1}(B)\right)$, and Lemma 5.4 identifies $B$ naturally with $\lim _{\lambda} B_{\lambda}$. Therefore it suffices to prove the lemma when $k=0$ and $n=1$.

We must prove:

(1) Every path component of $\mathrm{GL}_{1}(A)$ contains some element $\varphi_{\lambda, \infty}(b)$ for some $\lambda$ and some $b \in \mathrm{GL}_{1}\left(A_{\lambda}\right)$.

(2) If $b \in \mathrm{GL}_{1}\left(A_{\lambda}\right)$ and $\varphi_{\lambda, \infty}(b) \in \mathrm{GL}_{1}(A)_{\circ}$, then there exists $\mu \geq \lambda$ such that $\varphi_{\lambda, \mu}(b) \in \mathrm{GL}_{1}\left(A_{\mu}\right)_{\circ}$.

The key ingredient is that if $s \in A$ is invertible, then so is any $t \in A$ with $\|t-s\|<\left\|s^{-1}\right\|^{-1}$. We use this to prove the following claim: if $b \in A_{\lambda}$ and $\varphi_{\lambda, \infty}(b)$ is invertible, then there exists $\mu \geq \lambda$ such that $\varphi_{\lambda, \mu}(b)$ is invertible. Let $a=\varphi_{\lambda, \infty}(b)$. Set $\varepsilon_{0}=1 /(3\|a\|)$. Choose $\lambda_{0} \in \Lambda$ and $c_{0} \in A_{\lambda_{0}}$ such that $\left\|\varphi_{\lambda_{0}, \infty}\left(c_{0}\right)-a^{-1}\right\|<\varepsilon_{0}$. Then

$$
\left\|a \varphi_{\lambda_{0}, \infty}\left(c_{0}\right)-1\right\|<\varepsilon_{0}\|a\| \leq \frac{1}{3} .
$$

Choose $\lambda_{1} \geq \lambda, \lambda_{0}$. By Part (2) of the characterization of the direct limit, there exists $\mu_{0} \geq \lambda_{0}$ such that

$$
\left\|\varphi_{\lambda, \mu_{0}}(b) \varphi_{\lambda_{0}, \mu_{0}}\left(c_{0}\right)-1\right\|<\frac{2}{3} .
$$

In particular, $\varphi_{\lambda, \mu_{0}}(b) \varphi_{\lambda_{0}, \mu_{0}}\left(c_{0}\right)$ is invertible. Similarly, there is $\mu \geq \mu_{0}$ such that $\varphi_{\lambda_{0}, \mu}\left(c_{0}\right) \varphi_{\lambda, \mu}(b)$ is invertible. Then $\varphi_{\lambda_{0}, \mu}(b)$ is invertible. The claim is proved.

To prove (11), let $a \in \mathrm{GL}_{1}(A)$, and use density of $\bigcup_{\lambda \in \Lambda} \varphi_{\lambda, \infty}\left(A_{\lambda}\right)$ in $A$ to choose $\lambda_{0}$ and $b \in A_{\lambda_{0}}$ such that $\left\|\varphi_{\lambda_{0}, \infty}(b)-a\right\|<\left\|a^{-1}\right\|^{-1}$. Then the straight line path from $\varphi_{\lambda_{0}, \infty}\left(b_{0}\right)$ to $a$ is in $\mathrm{GL}_{1}(A)$. By the claim, there is $\lambda \geq \lambda_{0}$ such that $b=$ $\varphi_{\lambda_{0}, \lambda}\left(b_{0}\right)$ is invertible. 
To prove (22), let $b \in \mathrm{GL}_{1}\left(A_{\lambda}\right)$ satisfy $\varphi_{\lambda, \infty}(b) \in \mathrm{GL}_{1}(A)_{\circ}$. Let $t \mapsto a_{0}(t)$ be a continuous path from $\varphi_{\lambda, \infty}(b)$ to 1 in $\mathrm{GL}_{1}(A)$, defined for $t \in[1,2]$. Regard $t \mapsto a_{0}(t)$ as an invertible element $a \in C([1,2], A)$. By Lemma [5.4(2), we have $C([1,2], A)=\varliminf_{\longrightarrow} C\left([1,2], A_{\lambda}\right)$, so there is $\lambda_{0} \in \Lambda$ and $c_{0} \in C\left([1,2], A_{\lambda_{0}}\right)$ such that $\left\|\bar{\varphi}_{\lambda_{0}, \infty}\left(c_{0}\right)-a_{0}\right\|<\left\|a_{0}^{-1}\right\|^{-1}$. Without loss of generality $\lambda_{0} \geq \lambda$. Let $a \in C([0,3], A)$ be the concatenation of the constant path $\varphi_{\lambda, \infty}(b)$ on $[0,1]$, the path $a_{0}$ on $[1,2]$, and the constant path 1 on $[2,3]$. Let $c \in C\left([0,1], A_{\lambda_{0}}\right)$ be the concatenation of the straight line path from $\varphi_{\lambda, \lambda_{0}}(b)$ to $c_{0}(1)$ on $[0,1]$, the path $c_{0}$ on $[1,2]$, and the straight line path from $c_{0}(2)$ to 1 on $[2,3]$. Then also $\left\|\bar{\varphi}_{\lambda_{0}, \infty}(c)-a\right\|<\left\|a^{-1}\right\|^{-1}$. So $\bar{\varphi}_{\lambda_{0}, \infty}(c)$ is invertible. Applying the claim to the direct system $\left(C\left([0,3], A_{\lambda}\right)\right)_{\lambda \in \Lambda}$ (using Lemma 5.4(2) again), we find $\mu \geq \lambda_{0}$ such that $\bar{\varphi}_{\lambda_{0}, \mu}(c)$ is invertible. Since $\varphi_{\lambda_{0}, \mu}(c(3))=1$, we get $\varphi_{\lambda, \mu}(b)=\varphi_{\lambda_{0}, \mu}(c(0)) \in \mathrm{GL}_{1}\left(A_{\mu}\right)_{\circ}$.

Finally, we briefly consider direct limits of graded Lie algebras over $\mathbb{Q}$. Let $\Lambda$ be a directed set, for $\lambda \in \Lambda$ let $L_{\lambda}$ be a graded Lie algebra over $\mathbb{Q}$ as in Definition 4.24, and suppose, given graded Lie algebra maps, that $\psi_{\lambda, \mu}: L_{\lambda} \rightarrow L_{\mu}$ for $\lambda \leq \mu$ satisfying the usual coherence conditions. Define the direct limit $\lim _{\lambda} L_{\lambda}$ to be the graded space given, in each degree $n>0$, as the algebraic direct limit of $L_{\lambda}$ in degree $n$ with the bracket induced by the maps $\psi_{\lambda, \infty}: L_{\lambda} \rightarrow \lim _{\rightarrow} L_{\lambda}$. It is direct to check that $L$ with the induced bracket satisfies the axioms in Definition 4.24 . We need one easy result in this context.

Theorem 5.6. Let $\left(L_{\lambda}\right)_{\lambda \in \Lambda}$ be a direct system of graded Lie algebras over $\mathbb{Q}$ with graded Lie algebra structure maps $\psi_{\lambda, \mu}: L_{\lambda} \rightarrow L_{\mu}$. The direct limit $\lim _{\lambda} L_{\lambda}$ satisfies:

(1) Given a graded Lie algebra $L$ over $\mathbb{Q}$ and graded Lie algebra maps $\varphi_{\lambda}: L_{\lambda} \rightarrow$ $L$ satisfying $\varphi_{\mu} \circ \psi_{\lambda, \mu}=\varphi_{\lambda}$, there exists a unique graded Lie algebra map $\varphi: \lim _{\lambda} L_{\lambda} \rightarrow L$ satisfying $\varphi \circ \psi_{\lambda, \infty}=\varphi_{\lambda}$.

(2) If each $L_{\lambda}$ is abelian, then so is $\lim _{\lambda} L_{\lambda}$.

Proof. The proof of (1) is a direct consequence of the result for direct limits of groups. The proof of (2) is direct from the definition of the bracket in $\lim _{\longrightarrow} L_{\lambda}$.

We can now give the proof of our first main result.

Proof of Theorem 1. By Theorem 3.2, it suffices to prove the rational H-equivalence for algebras of the form $C(X)$, with $X$ compact Hausdorff, and to prove naturality for unital homomorphisms between such algebras, that is, for continuous maps (in the opposite direction) of compact Hausdorff spaces.

So let $X$ be compact Hausdorff. By Theorem 10.1 in Chapter X of 12, there exists a directed set $\Lambda$ and an inverse system $\left(X_{\lambda}\right)_{\lambda \in \Lambda}$ of finite CW complexes and continuous maps $f_{\lambda, \mu}: X_{\mu} \rightarrow X_{\lambda}$ for $\lambda \leq \mu$ with $X \approx \lim _{\lambda} X_{\lambda}$. We then obtain a direct system of $C^{*}$-algebras $\left(C\left(X_{\lambda}\right)\right)_{\lambda \in \Lambda}$ with maps $\overleftarrow{\varphi}_{\lambda, \mu}: C\left(X_{\lambda}\right) \rightarrow C\left(X_{\mu}\right)$ induced by $f_{\lambda, \mu}$. The maps $X \rightarrow X_{\lambda}$ determine an isomorphism of $C^{*}$-algebras $\lim _{\lambda} C\left(X_{\lambda}\right) \rightarrow C(X)$ because $Y \mapsto C(Y)$ is a contravariant equivalence of cate-

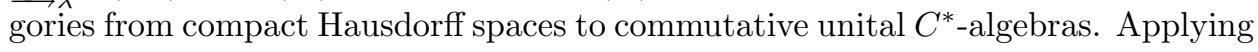
Theorem 5.5 and restricting ourselves to the identity components, we obtain isomorphisms $\lim _{\lambda} \pi_{k}\left(\mathrm{GL}_{n}\left(C\left(X_{\lambda}\right)\right)_{\circ}\right) \cong \pi_{k}\left(\mathrm{GL}_{n}(C(X))_{\circ}\right)$ for $k>0$. Since the tensor product commutes with direct limits, we obtain

$$
\underset{\lambda}{\lim } \pi_{k}\left(\mathrm{GL}_{n}\left(C\left(X_{\lambda}\right)\right)_{\circ}\right) \otimes \mathbb{Q} \cong \pi_{k}\left(\mathrm{GL}_{n}(C(X))_{\circ}\right) \otimes \mathbb{Q} .
$$


Set $V_{k, j}^{\lambda}=H^{2 j-1-k}\left(X_{\lambda} ; \mathbb{Q}\right)$ and let

$$
H\left(f_{\lambda, \mu}\right): \bigoplus_{j=1}^{n} V_{k, j}^{\lambda} \rightarrow \bigoplus_{j=1}^{n} V_{k, j}^{\mu}
$$

be the map induced on rational cohomology groups by $f_{\lambda, \mu}$. Since each $\operatorname{Max}\left(C\left(X_{\lambda}\right)\right)$ $\approx X_{\lambda}$ is a finite complex, Theorem 5.1 applies to give a commutative diagram

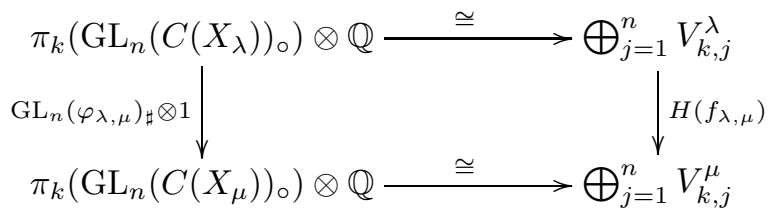

for each $k>0$. By the continuity of Čech cohomology (Theorem 3.1 in Chapter X of [12]; see page 110 for the definition of $\left.\mathcal{G}_{R}\right), \lim _{\lambda} V_{k, j}^{\lambda} \cong \check{H}^{2 j-1-k}(X ; \mathbb{Q})=\check{V}_{k, j}$. We conclude that

$$
\pi_{k}\left(\mathrm{GL}_{n}(C(X))_{\circ}\right) \otimes \mathbb{Q} \cong \bigoplus_{j=1}^{n} \check{V}_{k, j}
$$

Naturality is clear.

It remains to prove the rational $\mathrm{H}$-equivalence and show that $\mathrm{GL}_{n}(C(X))_{\text {。 }}$ is rationally homotopy-abelian. Consider the direct system $\left(\pi_{*}\left(\mathrm{GL}_{n}\left(C\left(X_{\lambda}\right)\right)_{\circ}\right) \otimes \mathbb{Q}\right)_{\lambda \in \Lambda}$ of graded Lie algebras over $\mathbb{Q}$ with structure maps

$$
\mathrm{GL}_{n}\left(\varphi_{\lambda, \mu}\right) \otimes 1: \pi_{*}\left(\mathrm{GL}_{n}\left(C\left(X_{\lambda}\right)\right)_{\circ}\right) \otimes \mathbb{Q} \rightarrow \pi_{*}\left(\mathrm{GL}_{n}\left(C\left(X_{\mu}\right)\right)_{\circ}\right) \otimes \mathbb{Q} .
$$

By Theorems 5.5 and 5.6(1) there is a graded Lie algebra isomorphism

$$
\underset{\lambda}{\lim } \pi_{*}\left(\mathrm{GL}_{n}\left(C\left(X_{\lambda}\right)\right)_{\circ}\right) \otimes \mathbb{Q} \cong \pi_{*}\left(\mathrm{GL}_{n}(C(X))_{\circ}\right) \otimes \mathbb{Q} .
$$

By Theorems 5.1 and $4.25(3)$, each $\pi_{*}\left(\mathrm{GL}_{n}\left(C\left(X_{\lambda}\right)\right)_{\circ}\right) \otimes \mathbb{Q}$ is abelian. Therefore $\pi_{*}\left(\mathrm{GL}_{n}(C(X))_{\circ}\right) \otimes \mathbb{Q}$ is abelian by Theorem $5.6(2)$. The space $\mathrm{GL}_{n}(C(X))$ is homotopy equivalent to a CW complex (Theorem[2.8(5)). So we may apply (3) $\Longrightarrow$ (2) and (3) $\Longrightarrow$ (4) of Theorem 4.25

Corollary 5.7. Let $A$ be a commutative unital Banach algebra and let $n>1$. Let $i: \mathrm{GL}_{n-1}(A) \circ \rightarrow \mathrm{GL}_{n}(A)$ 。 denote the inclusion. Let $\check{V}_{h, j}=\check{H}^{2 j-1-h}(\operatorname{Max}(A) ; \mathbb{Q})$. Then, after the identifications

$$
\pi_{k}\left(\mathrm{GL}_{n-1}(A)_{\circ}\right) \otimes \mathbb{Q} \cong \bigoplus_{j=1}^{n-1} \check{V}_{k, j} \quad \text { and } \quad \pi_{k}\left(\mathrm{GL}_{n}(A)_{\circ}\right) \otimes \mathbb{Q} \cong \bigoplus_{j=1}^{n} \check{V}_{k, j}
$$

for each $k \geq 1$ given by Theorem 1 , the map

$$
i_{\sharp} \otimes 1: \pi_{k}\left(\mathrm{GL}_{n-1}(A)_{\circ}\right) \otimes \mathbb{Q} \rightarrow \pi_{k}\left(\mathrm{GL}_{n}(A)_{\circ}\right) \otimes \mathbb{Q}
$$

corresponds to the inclusion of vector spaces

$$
\bigoplus_{j=1}^{n-1} \check{V}_{k, j} \hookrightarrow \bigoplus_{j=1}^{n} \check{V}_{k, j}
$$


Proof. As in the proof of Theorem 1 above, we may assume $A=C(X)$ and write

$$
\pi_{k}\left(\mathrm{GL}_{n-1}(A)_{\circ}\right) \otimes \mathbb{Q}=\underset{\lambda}{\lim } \pi_{k}\left(\mathrm{GL}_{n-1}\left(C\left(X_{\lambda}\right)\right)_{\circ}\right) \otimes \mathbb{Q}
$$

and

$$
\pi_{k}\left(\mathrm{GL}_{n}(A)_{\circ}\right) \otimes \mathbb{Q}=\underset{\lambda}{\lim } \pi_{k}\left(\mathrm{GL}_{n-1}\left(C\left(X_{\lambda}\right)\right)_{\circ}\right) \otimes \mathbb{Q}
$$

where each $X_{\lambda}$ is a finite complex. Now use naturality with respect to $n$ in Theorem [5.1]

We can now deduce Theorem 2 ,

Proof of Theorem 2. Consider the long exact sequence of Theorem 2.10 as far as the last abelian term $\pi_{1}\left(\mathrm{GL}_{n}(A)\right)$. In the groups $\pi_{k}(-)$ for $k \geq 1$, we may replace each space by the component containing the basepoint, obtaining

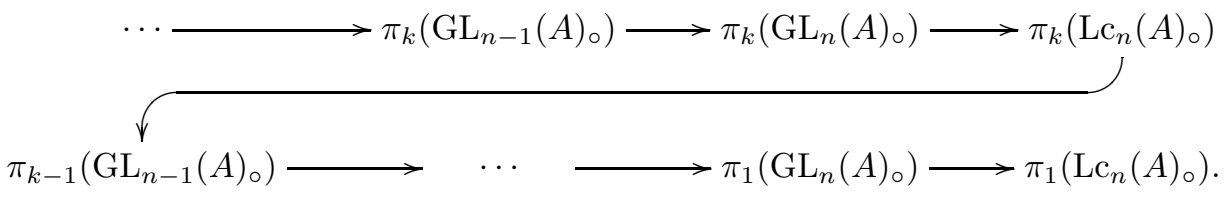

Tensor with $\mathbb{Q}$. Applying Corollary [5.7, we compute

$$
\pi_{k}\left(\operatorname{Lc}_{n}(A)_{\circ}\right) \otimes \mathbb{Q} \cong \check{H}^{2 n-1-k}(\operatorname{Max}(A) ; \mathbb{Q})
$$

for $k>1$, as needed.

We conclude with some remarks and a result concerning the rational homotopy type of the space of last columns for a commutative unital Banach algebra. By Theorem 3.6, we have $\operatorname{Lc}_{n}(A)_{\circ} \simeq F\left(\operatorname{Max}(A), S^{2 n-1}\right)_{\circ}$. Thus $\operatorname{Lc}_{n}(A)_{\circ}$ is a nilpotent CW complex when $\operatorname{Max}(A)$ is a finite complex by Theorem 4.18, Using Example 4.12 and Theorem 4.20, we conclude in this case that $\operatorname{Lc}_{n}(A)$ 。 is a rational $\mathrm{H}$-space. When $\operatorname{Max}(A)$ is not a finite complex, there is no guarantee that $\operatorname{Lc}_{n}(A)$ 。 is a nilpotent space (although we know of no counterexamples), and we cannot discuss its rational homotopy type. However, it follows from Theorem 2.8(5) that $\mathrm{Lc}_{n}(A)$ 。 has the homotopy type of a $\mathrm{CW}$ complex and thus admits a universal cover. Our last result describes the rational homotopy type of the universal cover of $\operatorname{Lc}_{n}(A)_{\circ}$ in the general case of a commutative unital Banach algebra. We need one final result from rational homotopy theory.

Lemma 5.8. Let $p: E \rightarrow B$ be a map of nilpotent $C W$ complexes inducing a surjection on rational homotopy groups. If $E$ is a rational $H$-space, then $B$ is one also.

Proof. This result is well-known in rational homotopy theory and easy to prove using minimal models. Since we have avoided their use thus far, we give a proof which does not use minimal models. Our argument adapts one given in 26; we reproduce it here for the sake of completeness.

As usual, we assume that the basepoint of each CW H-space $(G, \mu)$ is the identity. In addition, following Theorem III.4.7 of [33], we assume that the identity $e_{G}$ is strict, in the sense that $\mu\left(x, e_{G}\right)=\mu\left(e_{G}, x\right)=x$ for all $x \in G$.

Write $\pi_{*}\left(B_{\mathbb{Q}}\right)=\bigoplus_{i \in J} V_{i}$ with each $V_{i}$ a 1-dimensional rational vector space concentrated in degree $n_{i}$. For each $i$, pick a basis element $\alpha_{i}$ of $V_{i}$. Since $\left(p_{\mathbb{Q}}\right)_{\#}: \pi_{*}\left(E_{\mathbb{Q}}\right)$ $\rightarrow \pi_{*}\left(B_{\mathbb{Q}}\right)$ is surjective, we may choose $\beta_{i} \in \pi_{n_{i}}\left(E_{\mathbb{Q}}\right)$ such that $\left(p_{\mathbb{Q}}\right) \#\left(\beta_{i}\right)=\alpha_{i}$ for 
each $i$. If $n_{i}$ is odd, then $K\left(\mathbb{Q}, n_{i}\right) \simeq S_{\mathbb{Q}}^{n_{i}}$, and so we may regard $\beta_{i}$ as a map $\gamma_{i}: K\left(\mathbb{Q}, n_{i}\right) \rightarrow E_{\mathbb{Q}}$. Now suppose that $n_{i}$ is even. Then $K\left(\mathbb{Q}, n_{i}\right) \simeq \Omega \Sigma S_{\mathbb{Q}}^{n_{i}}$. For any space $X$, let $\varepsilon_{X}: X \rightarrow \Omega \Sigma X$ be the adjoint of the suspension of the identity map of $X$. This map is natural in $X$. Since $E_{\mathbb{Q}}$ is an H-space, by Theorem 8.14 of [28] there is a retraction $r: \Omega \Sigma E_{\mathbb{Q}} \rightarrow E_{\mathbb{Q}}$ of $\varepsilon_{E_{\mathbb{Q}}}$. Using this retraction, we have a commutative diagram

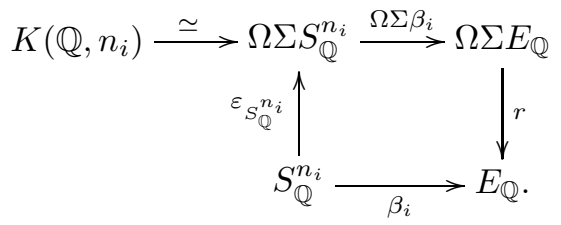

Now set $\gamma_{i}=r \circ \Omega \Sigma \beta_{i}: K\left(\mathbb{Q}, n_{i}\right) \rightarrow E_{\mathbb{Q}}$.

Choose a total order $\leq$ on $J$. For each finite subset $F \subset J$, set

$$
K_{F}=\prod_{i \in F} K\left(\mathbb{Q}, n_{i}\right) .
$$

We define a map $\alpha_{F}: K_{F} \rightarrow B_{\mathbb{Q}}$ as follows. Write $F=\left\{i_{1}, \ldots, i_{r}\right\}$ with $i_{1}<\cdots<$ $i_{r}$. Then, using $\cdot$ for the multiplication in $E_{\mathbb{Q}}$, set

$$
\alpha_{F}\left(x_{1}, \ldots, x_{r}\right)=p_{\mathbb{Q}}\left(\left(\left(\gamma_{i_{1}}\left(x_{1}\right) \cdot \gamma_{i_{2}}\left(x_{2}\right)\right) \cdot \gamma_{i_{3}}\left(x_{3}\right)\right) \cdots \cdots \gamma_{i_{r}}\left(x_{r}\right)\right) .
$$

The induced homomorphism on homotopy groups restricts to the inclusion $V_{i} \rightarrow$ $\pi_{*}\left(B_{\mathbb{Q}}\right)$ on each summand of $\pi_{*}\left(K_{F}\right) \cong \prod_{i \in F} V_{i}$.

If $F_{1} \subset F_{2} \subset J$ are finite sets, there is an obvious map $k_{F_{1}, F_{2}}: K_{F_{1}} \rightarrow K_{F_{2}}$, obtained by viewing $K_{F_{1}}$ as the set of elements $x \in K_{F_{2}}$ such that $x_{i}$ is the basepoint of $K\left(\mathbb{Q}, n_{i}\right)$ for $i \in F_{2} \backslash F_{1}$. Since the basepoints are the identities and the identities are strict, one checks that $\alpha_{F_{2}} \circ k_{F_{1}, F_{2}}=\alpha_{F_{1}}$ for $F_{1} \subset F_{2} \subset J$. Accordingly, we obtain a map $\alpha$ : $\lim _{F \subset J} K_{F} \rightarrow B_{\mathbb{Q}}$, which moreover induces an isomorphism on homotopy groups and hence is a homotopy equivalence. The H-space structures on the Eilenberg-Mac Lane spaces give obvious H-space structures on the products $K_{F}$, and the maps $k_{F_{1}, F_{2}}$ respect these structures and the associated homotopies. So $\underline{\lim }_{F \subset J} K_{F}$ is an H-space, and thus $B_{\mathbb{Q}}$ is also an H-space.

Theorem 5.9. Let $A$ be commutative unital Banach algebra. Let $\widetilde{\mathrm{Lc}_{n}(A)}$ 。 denote the universal cover of $\operatorname{Lc}_{n}(A)_{\circ}$. If $\operatorname{Max}(A)$ is a finite complex, then $\operatorname{Lc}_{n}(A)$ 。 is a rational $H$-space. In general, $\widetilde{\mathrm{Lc}_{n}(A)}$ 。 is a rational $H$-space.

Proof. When $\operatorname{Max}(A)$ is a finite complex the result is a consequence of Theorem 4.18, Example 4.12 and Theorem 4.20, as argued before the statement of Lemma 5.8. For the general case, observe that, by Theorem 2.10 and Corollary 5.7 . the map $\widetilde{\mathrm{GL}_{n}(A)}$ 。 $\widetilde{\mathrm{Lc}_{n}(A)}$ 。 induced on universal covers by the natural map $\mathrm{GL}_{n}(A)_{\circ} \rightarrow \mathrm{Lc}_{n}(A)$ 。 is a surjection on rational homotopy groups. $\mathrm{As}_{\mathrm{GL}_{n}(A)}$ 。 is a topological group, the result follows from Lemma 5.8.

\section{ACKNOWLEDGEMENT}

The authors would like to thank Daniel Isaksen and Jim Stasheff for helpful discussions. 


\section{REFERENCES}

[1] J. F. Adams, On the non-existence of elements of Hopf invariant one, Ann. Math. 72 (1960), 20-104. MR 25:4530

[2] M. F. Atiyah and R. Bott, The Yang-Mills equations over Riemann surfaces, Philos. Trans. Roy. Soc. London Ser. A 308 (1983), 523-615. MR85k:14006

[3] I. Berstein and T. Ganea, Homotopical nilpotency, Illinois J. Math. 5 (1961), 99-130. MR23:A3573

[4] F. F. Bonsall and J. Duncan, Complete normed algebras, Ergebnisse der Mathematik und ihrer Grenzgebiete, vol. 80, Springer-Verlag, New York, Heidelberg, 1973. MR54:11013

[5] A. Borel, Topology of Lie groups and characteristic classes, Bull. Amer. Math. Soc. 61 (1955), 397-432. MR17:282b

[6] L. G. Brown, P. Green, and M. A. Rieffel, Stable isomorphism and strong Morita equivalence of $C^{*}$-algebras, Pacific J. Math. 71 (1977), 349-363. MR57:3866

[7] G. Corach and A. R. Larotonda, Stable range in Banach algebras, J. Pure Appl. Algebra 32 (1984), 289-300. MR86g:46070

[8] — A stabilization theorem for Banach algebras, J. Algebra 101 (1986), 433-449. MR $87 \mathrm{~h}: 46103$

[9] C. J. Curjel, On the H-space structures of finite complexes, Comment. Math. Helv. 43 (1968), 1-17. MR37:6929

[10] A. M. Davie, Homotopy in Fréchet algebras, Proc. London Math. Soc. (3) 23 (1971), 31-52. MR $45: 5756$

[11] J. Dixmier and A. Douady, Champs continus d'espaces hilbertiens et de $C^{*}$-algèbres, Bull. Soc. Math. France 91 (1963), 227-284. MR:29:485

[12] S. Eilenberg and N. E. Steenrod, Foundations of algebraic topology, Princeton University Press, Princeton, 1952. MR,14:398b

[13] Y. Félix, S. Halperin, and J.-C. Thomas, Rational homotopy theory, Graduate Texts in Mathematics, vol. 205, Springer-Verlag, New York, 2001. MR2002d:55014

[14] D. Handelman, $K_{0}$ of von Neumann and $A F C^{*}$ algebras, Quart. J. Math. Oxford Ser. (2) 29 (1978), 427-441. MR81c:46049

[15] P. Hilton, G. Mislin, and J. Roitberg, Localization of nilpotent groups and spaces, NorthHolland Publishing Co., Amsterdam, 1975, North-Holland Mathematics Studies, No. 15, Notas de Matemática, No. 55 [Notes on Mathematics, No. 55]. MR 57:17635

[16] I. M. James, On H-spaces and their homotopy groups, Quart. J. Math. Oxford Ser. (2) 11 (1960), 161-179. MR24:A2966

[17] J. L. Kelley, General topology, Van Nostrand Reinhold, New York, Cincinnati, Toronto, London, Melbourne, 1955. MR16:1136c

[18] A. T. Lundell and S. Weingram, The topology of CW complexes, Van Nostrand Reinhold Company, New York, 1969.

[19] R. J. Milgram, The bar construction and abelian $H$-spaces, Illinois J. Math 11 (1967), 242250. MR 34:8404

[20] J. Milnor, The geometric realization of a semi-simplicial complex, Ann. Math. 65 (1957), 357-362. MR 18:815d

[21] (1959), 272-280. MR20:6700

[22] G. J. Murphy, $C^{*}$-algebras and operator theory, Academic Press, Boston, San Diego, New York, London, Sydney, Tokyo, Toronto, 1990. MR91m:46084

[23] N. C. Phillips, Equivariant K-theory and freeness of group actions on $C^{*}$-algebras, SpringerVerlag Lecture Notes in Math. no. 1274, Springer-Verlag, Berlin, Heidelberg, New York, London, Paris, Tokyo, 1987. MR.89k:46086

[24] M. A. Rieffel, Dimension and stable rank in the $K$-theory of $C^{*}$-algebras, Proc. London Math. Soc. (3) 46 (1983), 301-333. MR84g:46085

[25] - The homotopy groups of the unitary groups of noncommutative tori, J. Operator Theory 17 (1987), 237-254. MR88f:22018

[26] H. Scheerer, On rationalized $H$ - and co-H-spaces. With an appendix on decomposable $H$ - and co-H-spaces, Manuscripta Math. 51 (1985), 63-87. MR88k:55007

[27] E. H. Spanier, Algebraic topology, McGraw-Hill, New York, San Francisco, St. Louis, Toronto, London, Sydney, 1966. MR 35:1007 
[28] J. Stasheff, H-spaces from a homotopy point of view, Lecture Notes in Mathematics, vol. 161, Springer-Verlag, Berlin, New York, 1970. MR42:5261

[29] J. L. Taylor, Banach algebras and topology, Algebras in analysis (Proc. Instructional Conf. and NATO Advanced Study Inst., Birmingham, 1973), Academic Press, London, 1975, pp. 118-186. MR:54:5837

[30] A. B. Thom, Connective E-theory and bivariant homology for $C^{*}$-algebras, Ph.D. thesis, U. Münster, 2003. math.uni-muenster.de/inst/sfb/about/publ/thom.html

[31] R. Thom, L'homologie des espaces fonctionnels, Colloque de topologie algébrique, Louvain, 1956, Georges Thone, Liège, 1957, pp. 29-39. MR:19:669h

[32] K. Thomsen, Nonstable K-theory for operator algebras, K-Theory 4 (1991), 245-267. MR92h:46102

[33] G. W. Whitehead, Elements of homotopy theory, Graduate Texts in Mathematics, vol. 61, Springer-Verlag, New York, 1978. MR:80b:55001

Department of Mathematics, Cleveland State University, Cleveland, Ohio 44115

E-mail address: G.Lupton@csuohio.edu

Department of Mathematics, University of Oregon, Eugene, Oregon 97403-1222

E-mail address: ncp@darkwing.uoregon.edu

Department of Mathematics, Wayne State University, Detroit, Michigan 48202

E-mail address: claude@math.wayne.edu

Department of Mathematics, Saint Joseph's University, Philadelphia, Pennsylvania 19131

E-mail address: smith@sju.edu 